\begin{tabular}{|c|l|}
\hline Title & Singularity theory of smooth mappings and its applications:a survey for non-specialists \\
\hline Author(s) & IZUMIY A, Shy uichi \\
\hline Citation & Hokkaido University technical report series in mathematics, 108, 1 \\
\hline Issue Date & 2006-01-01 \\
\hline DOI & 10.14943/8082 \\
\hline Doc URL & http://hdl.handle.net/2115/8466; http://eprints3.math.sci.hokudai.ac.jp/1525/ \\
\hline Type & bulletin (article) \\
\hline Nile Information & Sydney proc.pdf \\
\hline
\end{tabular}

Instructions for use 


\title{
Singularity theory of smooth mappings and its applications: a survey for non-specialists
}

\author{
Shyuichi IZUMIYA
}

April 10, 2006

\begin{abstract}
This is an elementary survey on the results of the singularity theory of smooth mapping and its applications.
\end{abstract}

\section{Introduction: Elementary calculus}

There are two roots of the theory of singularities of smooth mappings. One is the Morse theory another is the theory of immersions and embeddings of manifolds. However, the both theory are originated by the theory of smooth functions of one-variable. Here we start to review some results on functions of one-variable from the elementary calculus. In the course of the elementary calculus, one of the most exciting results is that we can solve the extremal problem as an application of differential calculus. By using the method of differential calculus, we can recognize the shape of graphs of smooth functions of one-variable. How can we recognize the shape of the graph? The algorithm is as follows:

1) Calculate the first derivative $f^{\prime}(x)$ of $f(x)$ and find a point $x_{0}$ such that $f^{\prime}\left(x_{0}\right)=0$.

2) Calculate the second derivative $f^{\prime \prime}(x)$, then

$$
\text { the point } x_{0} \text { is }\left\{\begin{array}{c}
\text { minimal if } f^{\prime \prime}\left(x_{0}\right)>0 \\
\text { maximal if } f^{\prime \prime}\left(x_{0}\right)<0
\end{array}\right. \text {. }
$$

We say that $x_{0}$ is a critical point (or, singular point) of $f(x)$ if $f^{\prime}\left(x_{0}\right)=0$. The shape of the graph $y=f(x)$ is drastically changing around the singular point. Therefore the singular point of $f(x)$ gives a crucial information on the shape of the graph $y=f(x)$. Moreover we call $x_{0}$ a degenerate singular point if $f^{\prime}\left(x_{0}\right)=f^{\prime \prime}\left(x_{0}\right)=0$. By the above fact, the non-degenerate singular points are maximal points or minimal points. However, we can easily recognize the local shape of the graph even if $x_{0}$ is a degenerate singular point. We say that $x_{0}$ is an $A_{k}$-type singular point if $f^{\prime}\left(x_{0}\right)=f^{\prime \prime}\left(x_{0}\right)=\cdots=f^{(k)}\left(x_{0}\right)=0$ and $f^{(k+1)}\left(x_{0}\right) \neq 0$. Suppose that $x_{0}$ is an $A_{k}$-type singular point, it is known that $x_{0}$ is minimal if $k$ is even and it is maximal if $k$ is odd. In fact, we have the following theorem:

2000 Mathematics Subject classification 58C28

Key Words and Phrases.

Partially supported by Grant-in-Aid for formation of COE

"Mathematics of Nonlinear Structure via Singularities" (Hokkaido University) 
Theorem 1.1 Let $f:(\mathbb{R}, 0) \longrightarrow \mathbb{R}$ be a $C^{\infty}$-function germ at 0 . Suppose that 0 is an $A^{k}$-type singular point, then there exists a local diffeomorphism germ $\phi:(\mathbb{R}, 0) \longrightarrow(\mathbb{R}, 0)$ such that

$$
f \circ \phi(x)= \pm x^{k+1}+f(0) .
$$

Proof. We give only an outline of the proof. Assume that $f(0)=0$. Since 0 is an $A_{k^{-}}$ type singularity, there exists a $C^{\infty}$-function germ $g_{k+1}(x)$ with $g_{k+1}(0) \neq 0$ such that $f(x)=$ $x^{k+1} g_{k+1}(x)$. If we define $\psi(x)=\left( \pm g_{k+1}(x)\right)^{\frac{1}{k+1}} x$, then we can show that $\psi$ is a diffeomorphism germ. We put $\phi=p s i^{-1}$, so that we have $f \circ \phi(x)= \pm x^{k+1}$.

Since the local diffeomorphism (diffeomorphism germ) preserves the local "shape" of the graph of function, the graph of the function with an $A_{k}$-type singular point is almost the same as the graph of $y= \pm x^{k+1}$ (see Fig. 1.1).

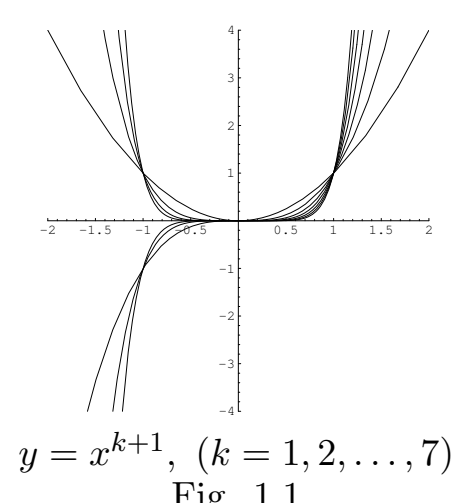

Fig. 1.1

On the other hand, the inverse function theorem is also a very exiting results of elementary calculus.

Theorem 1.2 Let $f:(\mathbb{R}, 0) \longrightarrow(\mathbb{R}, 0)$ be a $C^{\infty}$-function germ at 0 . Then 0 is a non-singular point (i.e., $f^{\prime}(0) \neq 0$ ) if and only if there exists a inverse function germ $f^{-1}:(\mathbb{R}, 0) \longrightarrow(\mathbb{R}, 0)$ of $f$.

Around a singular point of $f$, the inverse function $f^{-1}$ might be multi-valued. For example, we consider a function given by $f(x)=x^{3}-x$. In this case the "inverse function" is $x=y^{3}-y$, so that $y=f^{-1}(x)$ is 3 -valued function (cf., Fig. 1.2, 1.3).

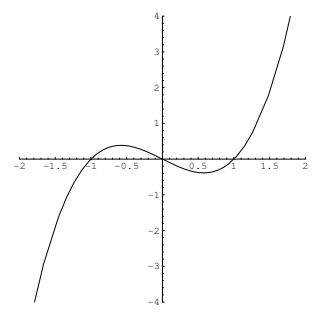

Fig. 1.2: $y=x^{3}-x$

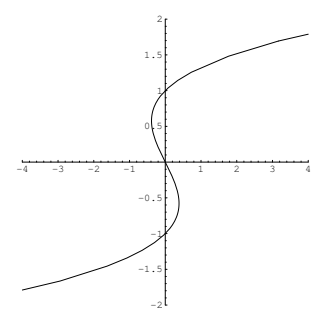

Fig. $1.3: x=y^{3}-y$

In this paper we describe how we can generalize these facts to the case for several variables. There are two directions of such generalizations: 
(1) Functions with several variables.

(2) Vector valued functions (smooth mappings).

The first case is related to the Morse theory and the second one is the embeddings or immersions theory of manifolds.

In this paper we assume that all mappings and manifolds are of class $C^{\infty}$ unless stated otherwise.

\section{Smooth functions of several variables}

We start to review the extremal problem of smooth functions of two variables $z=f(x, y)$. Like as the case for functions of one-variable, we have the following algorithm for solving the extremal problem:

(1) Find a singular point (critical point) $\left(x_{0}, y_{0}\right)$ (i.e., $\left.\partial f / \partial x\left(x_{0}, y_{0}\right)=\partial f / \partial y\left(x_{0}, y_{0}\right)=0\right)$.

(2) Consider the Hessian matrix

$$
\mathcal{H}(f)\left(x_{0}, y_{0}\right)=\left(\begin{array}{ll}
\frac{\partial^{2} f}{\partial x^{2}}\left(x_{0}, y_{0}\right) & \frac{\partial^{2} f}{\partial x \partial y}\left(x_{0}, y_{0}\right) \\
\frac{\partial^{2} f}{\partial x \partial y}\left(x_{0}, y_{0}\right) & \frac{\partial^{2} f}{\partial y^{2}}\left(x_{0}, y_{0}\right)
\end{array}\right)
$$

(3) Then

$$
\text { the point }\left(x_{0}, y_{0}\right) \text { is }\left\{\begin{array}{r}
\text { maximal or minimal if } \operatorname{det} \mathcal{H}(f)\left(x_{0}, y_{0}\right)>0 \\
\text { non-extremal if } \operatorname{det} \mathcal{H}(f)\left(x_{0}, y_{0}\right)<0
\end{array}\right. \text {. }
$$

If $\operatorname{det} \mathcal{H}(f)\left(x_{0}, y_{0}\right)=0$, the situation depends on the individual cases. For the singular point $\left(x_{0}, y_{0}\right)$ with $\operatorname{det} \mathcal{H}(f)\left(x_{0}, y_{0}\right) \neq 0$, the local shape of the graph $y=f(x, y)$ looks like $y=$ $\pm\left(x^{2}+y^{2}\right)$ or $y= \pm\left(x^{2}-y^{2}\right)$. (See Fig. 2.1 and Fig. 2.2). This is the reason why we can distinguish the point is extremal or not for the case $\operatorname{det} \mathcal{H}(f)\left(x_{0}, y_{0}\right) \neq 0$. The above arguments might be called the "local Morse theory" for functions with two variables.

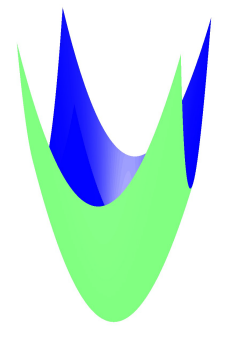

Fig. 2.1.

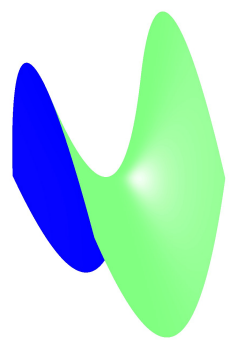

Fig. 2.2.

We can generalize the above arguments to functions with $n$-variables as follows: We say that $\boldsymbol{x}_{0} \in \mathbb{R}^{n}$ is a singular point (critical point) of a smooth function $f\left(x_{1}, \ldots, x_{n}\right)$ if $\partial f / \partial x_{i}\left(\boldsymbol{x}_{0}\right)=0$ for $i=1, \ldots, n$. In this case the Hessian matrix is a $n \times n$ matrix defined by

$$
\mathcal{H}(f)\left(\boldsymbol{x}_{0}\right)=\left(\frac{\partial^{2} f}{\partial x_{i} \partial x_{j}}\left(\boldsymbol{x}_{0}\right)\right) .
$$


A singular point $\boldsymbol{x}_{0}$ of $f\left(x_{1}, \ldots, x_{n}\right)$ is non-degenerate if det $\mathcal{H}(f)\left(\boldsymbol{x}_{0}\right) \neq 0$. For convenience, we use the notion of "germs" (i.e., function germs, map germs, set germs etc). The reader who does not know the notion of germs might consider the subjects (functions, maps, sets etc) around a point in locally. Then we have the following Morse lemma (cf., Milnor[55])

Theorem 2.1 (The Morse lemma) Let $0 \in \mathbb{R}^{n}$ be a non-degenerate singular point of $f\left(x_{1}, \ldots x_{n}\right)$. Then there exists a diffeomorphism germ $\phi:\left(\mathbb{R}^{n}, 0\right) \longrightarrow\left(\mathbb{R}^{n}, 0\right)$ such that

$$
f \circ \phi\left(x_{1}, \ldots, x_{n}\right)= \pm x_{1}^{2} \pm \cdots \pm x_{n}^{2}+f(0) .
$$

By the Morse lemma, we can recognize the shape of the graph $y=f\left(x_{1}, \ldots, x_{n}\right)$ around a nondegenerate singular point very well. If we consider the global situation, we consider smooth functions on manifolds. Locally the shape of the graph (i.e., the manifold) is well understood by the above reason. Moreover we can obtain global information by Morse functions (i.e., smooth functions with only non-degenerate singular points and the critical values are different). This is known as the Morse theory on manifolds.

We now arrange the terminology of the local theory of singularity for smooth functions. Let $f, g:\left(\mathbb{R}^{n}, 0\right) \longrightarrow \mathbb{R}$ be smooth function germs. We say that $f$ and $g$ are $\mathcal{R}^{+}$-equivalent if there exists a diffeomorphism germ $\phi:\left(\mathbb{R}^{n}, 0\right) \longrightarrow\left(\mathbb{R}^{n}, 0\right)$ and a real number $c$ such that $f \circ \phi\left(x_{1}, \ldots, x_{n}\right)+c=g\left(x_{1}, \ldots, x_{n}\right)$. Under this terminology, the Morse lemma asserts that the smooth function germ $f$ at the non-degenerate singular point 0 is $\mathcal{R}^{+}$-equivalent to a non-degenerate quadratic form. This means that the non-degenerate quadratic forms give the normal forms of functions with non-degenerate singular point.

On the other hand, we say that $0 \in \mathbb{R}^{n}$ is a degenerate singular point if $\partial f / \partial x_{i}(0)=$ $\operatorname{det}\left(\partial^{2} f / \partial x_{i} \partial x_{j}(0)=0 i, j=1, \ldots, n\right.$. The next question is as follows:

Problem: How can we study degenerate singular points?

In order to consider this problem, we introduce the following notion :

$$
\operatorname{corank}(f)(0)=n-\operatorname{rank} \mathcal{H}(f)(0) .
$$

We call it the corank of $f$ at 0 . A singular point 0 of $f$ is non-degenerate if and only if corank $(f)(0)=0$. We can interpret that the Morse lemma is a classification theorem on smooth functions around corank zero singular point. The following theorem is a stepping stone to the next development of the singularity theory of functions with several variables.

Theorem 2.2 (Thom's splitting lemma) Let $0 \in \mathbb{R}^{n}$ be a singular point of $f\left(x_{1}, \ldots, x_{n}\right)$ with corank $(f)(0)=r$. Then there exist a diffeomorphism germ $\phi:\left(\mathbb{R}^{n}, 0\right) \longrightarrow\left(\mathbb{R}^{n}, 0\right)$ and a smooth function germ $g\left(x_{1}, \ldots, x_{r}\right)$ at 0 such that

$$
f \circ \phi\left(x_{1}, \ldots x_{n}\right)=g\left(x_{1}, \ldots, x_{r}\right) \pm x_{r+1}^{2} \pm \cdots \pm x_{n}^{2}+f(0)
$$

and $\operatorname{rank} \mathcal{H}(g)(0)=0$.

We call $g$ a residual singularity of $f$. By the above theorem, it is enough to consider the residual singularity in order to classify the function germs by the $\mathcal{R}^{+}$-equivalence. If corank $(f)(0)=1$, the residual singularity $g$ is a smooth function of one-variable, so that we can classify $f$ by Theorem 1.1 . For the case corank $(f)(0) \geq 2$, we need the notion of unfoldings. Let $f:\left(\mathbb{R}^{n}, 0\right) \longrightarrow(\mathbb{R}, 0)$ be a smooth function germ. We say that a smooth function 
germ $F:\left(\mathbb{R}^{n} \times \mathbb{R}^{r}, 0\right) \longrightarrow(\mathbb{R}, 0)$ is an $r$-parameter unfolding of $f$ if $F(x, 0)=f(x)$. We denote $\mathcal{E}_{m}$ the local ring of smooth function germs $\left(\mathbb{R}^{m}, 0\right) \longrightarrow \mathbb{R}$ with the unique maximal ideal $\mathfrak{M}_{m}=\left\{h \in \mathcal{E}_{m} \mid h(0)=0\right\}$. Let $F, G:\left(\mathbb{R}^{n} \times \mathbb{R}^{r}, 0\right) \longrightarrow(\mathbb{R}, 0)$ be unfoldings. We say that $F$ and $G$ are $P$ - $\mathcal{R}^{+}$-equivalent if there exists a diffeomorphism germ $\Phi:\left(\mathbb{R}^{n} \times \mathbb{R}^{r}, 0\right) \longrightarrow\left(\mathbb{R}^{n} \times \mathbb{R}^{r}, 0\right)$ of the form $\Phi(x, u)=\left(\Phi_{1}(x, u), \phi(u)\right)$ and a function germ $h:\left(\mathbb{R}^{r}, 0\right) \longrightarrow \mathbb{R}$ such that $G(x, u)=F(\Phi(x, u))+h(u)$. For any $F_{1} \in \mathfrak{M}_{n+r}$ and $F_{2} \in \mathfrak{M}_{n^{\prime}+r}, F_{1}, F_{2}$ are said to be stably $P-\mathcal{R}^{+}$-equivalent if they become $P-\mathcal{R}^{+}$-equivalent after the addition to the arguments to $x_{i}$ of new arguments $y_{i}$ and to the functions $F_{i}$ of nondegenerate quadratic forms $Q_{i}$ in the new arguments (i.e., $F_{1}+Q_{1}$ and $F_{2}+Q_{2}$ are $P$ - $\mathcal{R}^{+}$-equivalent).

Let $F:\left(\mathbb{R}^{n} \times \mathbb{R}^{r}, 0\right) \longrightarrow(\mathbb{R}, 0)$ be an $r$-parameter unfolding of $f$. We say that $F$ is an $\mathcal{R}^{+}$-versal unfolding of if

$$
\mathcal{E}_{n}=J_{f}+\left\langle\frac{\partial F}{\partial u_{1}}\left|\mathbb{R}^{n} \times\{0\}, \ldots, \frac{\partial F}{\partial u_{r}}\right| \mathbb{R}^{n} \times\{0\}\right\rangle_{\mathbb{R}}+\langle 1\rangle_{\mathbb{R}}
$$

where

$$
J_{f}=\left\langle\frac{\partial f}{\partial x_{1}}, \ldots, \frac{\partial f}{\partial x_{n}}\right\rangle_{\mathcal{E}_{n}} .
$$

For classification for $\mathcal{R}^{+}$-versal unfolding, we need extra machinery such as "finite determinacy" and "versality theorem" etc. The reader who is interested in the detail of the story can refer to the books $([2,8,48])$. We only refer the following classification theorem which is called the Thom's seven elementary catastrophes.

Theorem 2.3 Let $f:\left(\mathbb{R}^{n}, 0\right) \longrightarrow(\mathbb{R}, 0)$ be a smooth function germ with

$$
1 \leq \mu(f)=\operatorname{dim}_{\mathbb{R}} \frac{\mathcal{E}_{n}}{J_{f}} \leq 5 .
$$

The $(\mu(f)-1)$-parameter $\mathcal{R}^{+}$-versal unfolding of $f$ is stably $P$ - $\mathcal{R}^{+}$-equivalent to one of the following unfoldings:

(1) $x_{1}^{3}+u_{1} x_{1}$,

(2) $\pm x_{1}^{4}+u_{1} x_{1}^{2}+u_{2} x_{1}$,

(3) $x_{1}^{5}+u_{1} x_{1}^{3}+u_{2} x_{1}^{2}+u_{3} x_{1}$,

(4) $\pm x_{1}^{6}+u_{1} x_{1}^{4}+u_{2} x_{1}^{3}+u_{3} x_{1}^{2}+u_{4} x_{1}$,

(5) $x_{1}^{3}+x_{2}^{3}+u_{1} x_{1} x_{2}+u_{2} x_{1}+u_{3} x_{2}$,

(6) $x_{1}^{3}-x_{1} x_{2}+u_{1}\left(x_{1}^{2}+x_{2}^{2}\right)+u_{2} x_{1}+u_{3} x_{2}$

(7) $\pm\left(x_{1}^{2} x_{2}+x_{2}^{4}\right)+u_{1} x_{1}^{2}+u_{2} x_{2}^{2}+u_{3} x_{1}+u_{4} x_{2}$.

We will return to this subject in $\S 4$ from the different (more sophisticated) view point.

\section{Singularities of smooth mappings}

The inverse function theorem (Theorem 1.2) asserts that the inverse function around the singular point is also a single valued smooth function. We now consider the vector valued version of the inverse function theorem. It can be formulated as the implicit function theorem (cf., 
[15], Theorem 2.4). Let $f: \mathbb{R}^{n} \longrightarrow \mathbb{R}^{p}$ be a smooth mapping. We consider the Jacobi matrix at $\boldsymbol{x} \in \mathbb{R}^{n}$ is the $p \times n$-matrix defined as follows:

$$
J_{f}(\boldsymbol{x})=\left(\frac{\partial f_{i}}{\partial x_{j}}(\boldsymbol{x})\right),
$$

where $f(\boldsymbol{x})=\left(f_{1}(\boldsymbol{x}), \ldots, f_{p}(\boldsymbol{x})\right)$ and $\boldsymbol{x}=\left(x_{1}, \ldots, x_{n}\right)$. As a corollary of the implicit function theorem, we have the following theorem:

Theorem 3.1 Let $f:\left(\mathbb{R}^{n}, 0\right) \longrightarrow\left(\mathbb{R}^{p}, 0\right)$ be a $C^{\infty}$-map germ. If rank $J_{f}(0)=\min (n, p)$, then there exist diffeomorphism germs $\phi:\left(\mathbb{R}^{n}, 0\right) \longrightarrow\left(\mathbb{R}^{n}, 0\right)$ and $\psi:\left(\mathbb{R}^{p}, 0\right) \longrightarrow\left(\mathbb{R}^{p}, 0\right)$ such that the following conditions hold:

(1) If $n \leq p$ then $\psi \circ f \circ \phi^{-1}\left(x_{1}, \ldots, x_{n}\right)=\left(x_{1}, \ldots, x_{n}, 0, \ldots, 0\right)$.

(2) If $n>p$ then $\psi \circ f \circ \phi^{-1}\left(x_{1}, \ldots, x_{n}\right)=\left(x_{1}, \ldots, x_{p}\right)$.

We say that $f$ is an immersion at 0 if the condition (1) in the above theorem holds and a submersion if the condition (2) holds. We also say that 0 is a singular point of $f$ if rank $J_{f}(0)<$ $\min (n, p)$. We have the following typical examples of singular points:
(a) $f:\left(\mathbb{R}^{2}, 0\right) \longrightarrow\left(\mathbb{R}^{3}, 0\right) ; f(x, y)=\left(x^{2}, y, x y\right)$,
(b) $f:\left(\mathbb{R}^{2}, 0\right) \longrightarrow\left(\mathbb{R}^{2}, 0\right) ; f(x, y)=\left(x, y^{2}\right)$,
(c) $f:\left(\mathbb{R}^{2}, 0\right) \longrightarrow\left(\mathbb{R}^{2}, 0\right) ; f(x, y)=\left(x, y^{3}+x y\right)$.

Example (a) The germ of example (a) is called the Whitney's umbrella (or, cross cap). The Jacobian matrix is given by

$$
J_{f}(x, y)=\left(\begin{array}{cc}
2 x & 0 \\
0 & 1 \\
y & x
\end{array}\right)
$$

It follows that the singular point is the origin $(0,0)$. The image of this map-germ is depicted in Fig. 3.1.

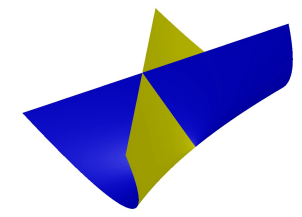

Whitney's umbrella

Fig. 3.1.

This map germ was discovered by Whitney[78] in the process of the research of immersions and embeddings of manifolds. Actually, Whitney has shown the following theorem[78].

Theorem 3.2 (Whitney's immersion theorem) Let $N$ be an n-dimensional manifold. The set of immersions

$$
\operatorname{Imm}\left(N, \mathbb{R}^{2 n}\right)=\left\{i: N \longrightarrow \mathbb{R}^{2 n} \mid \operatorname{rank} d i_{x}=n \text { at any } x \in N\right\}
$$


is an open and dense subset of the space of all $C^{\infty}$-mappings $C^{\infty}\left(N, \mathbb{R}^{2 n}\right)$ equipped with Whitney $C^{\infty}$-topology.

The natural question after the above immersion theorem was proven has been given as follows:

Question How about the set $\operatorname{Imm}\left(N, \mathbb{R}^{2 n-1}\right)$ ?

The answer is that the set of immersion is not dense in $C^{\infty}\left(N, \mathbb{R}^{2 n-1}\right)$. Whitney[78] discovered that there are $C^{\infty}$ - mapping which are written

$$
f\left(x_{1}, \ldots, x_{n}\right)=\left(x_{1}^{2}, x_{2}, \ldots, x_{n}, x_{1} x_{2}, \ldots x_{1} x_{n}\right)
$$

under suitable local coordinates on $N$ and $\mathbb{R}^{2 n-1}$ around a singular point. The above singular point cannot be changed under any small perturbation of $f$. Such a singular point is called a stable singular point. The case $n=2$ is the Whitney's umbrella. This is the first stable singular point of $C^{\infty}$-mappings appearing in the history of Singularity theory of smooth mappings.

Example (b) The germ of example (b) is called the fold. The Jacobian matrix is given by

$$
J_{f}(x, y)=\left(\begin{array}{cc}
1 & 0 \\
0 & 2 y
\end{array}\right)
$$

It follows that the set of the singular points is the $x$-axis $(x, 0)$. The critical value set is also the $X$-axis $(X, 0)$. We describe how we can observe the singularities. Define a mapping

$$
G_{f}:\left(\mathbb{R}^{2}, 0\right) \longrightarrow\left(\mathbb{R}^{3}, 0\right) ; G_{f}(x, y)=\left(x, y^{2}, y\right) .
$$

If we consider the canonical projection $\pi(X, Y, Z)=(X, Y)$, then we have $f(x, y)=\pi \circ G_{f}(x, y)$. The map germ $G_{f}$ is an embedding, so that the image is a surface without singular points. This surface can be considered as the graph of $f$. We can draw the picture of the image of $G_{f}$ in Fig.3.2.

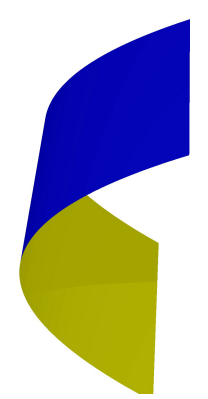

fold

Fig. 3.2.

Example (c) The germ of example (c) is called the cusp(or pleat). The Jacobian matrix is given by

$$
J_{f}(x, y)=\left(\begin{array}{cc}
1 & 0 \\
y & 3 y^{2}+x
\end{array}\right)
$$

It follows that the set of the singular points is the parabola $\left(-3 y^{2}, y\right)$ (cf., Fig.3.3). The critical value set is also the $3 / 2$-cusp $\left(-3 y^{2},-2 y^{3}\right)$ (cf., Fig. 3.3). 


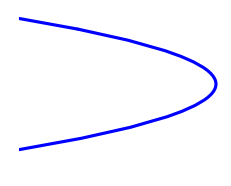

parabola

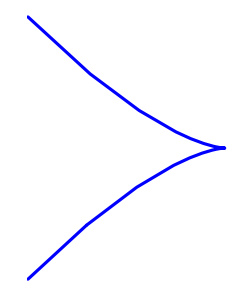

$3 / 2$-cusp

Fig. 3.3

We also define a mapping

$$
G_{f}:\left(\mathbb{R}^{2}, 0\right) \longrightarrow\left(\mathbb{R}^{3}, 0\right) ; G_{f}(x, y)=\left(x, y^{3}+x y, y\right),
$$

then we have $f(x, y)=\pi \circ G_{f}(x, y)$. We can also draw the picture of the image of $G_{f}$ in Fig.3.3. which looks like a pleat.

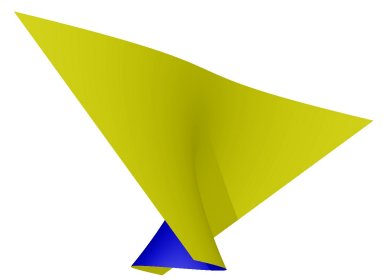

pleat

Fig. 3.4.

In both cases, $G_{f}$ can be considered as a unfolding of the folded singularities. This is the germ of the notion of unfoldings due to Thom. For folds and pleats, there is a theorem of Whitney.

Theorem 3.3 (Whitney's plane to plane mappings) Let $N, P$ be two dimensional manifolds.

$$
\mathcal{O}=\left\{f \in C^{\infty}(N, P) \mid f \text { has only folds or cusps as singularities }\right\}
$$

is an open dense subset of $C^{\infty}(N, P)$.

Here, $C^{\infty}(N, P)$ is the space of all $C^{\infty}$-mappings from $N$ to $P$, equipped with Whitney $C^{\infty}$-topology.

For the detailed description, we only refer books $[15,48]$. Here, we only remark that the fold and the cusp are "stable" singularities. Therefore, we might say that $C^{\infty}$-mappings with only folds or cusps are "generic" in the space of $C^{\infty}$-mappings between two dimensional manifolds. For general dimensional manifolds, Thom proposed some problems (for example, see [74]. Around 1970, J. Mather solved the main part of Thom's problems (see the celebrated series of Mather's 
papers $[49,50,51,52,53,54,14])$. However, there are still many open problems in the topological theory on singularities of smooth mappings (cf., du Plessis-Wall [64]).

On the other hand, if we consider a surface $N \subset \mathbb{R}^{3}$ and an orthogonal projection $\pi$ : $\mathbb{R}^{3} \longrightarrow P$ on to a plane $P$ in $\mathbb{R}^{3}$, then the restriction $f=\pi \mid N: N \longrightarrow P$ can be considered as a "camera". By Theorem 3.3, the contour of $f(N)$ looks like "folds" or "cusps". This fact leads the "Mathematical theory of solid shape" (see, Koenderink[44]). We will come back to this topic later.

\section{Lagrangian and Legendrian singularities}

In this section we give a brief review on the theory of Lagrangian and Legendrian singularities due to $[2,79]$ which are the geometric version of the theory of Thom's elementary catastrophes.

First, we describe the theory of Lagrangian singularities. We consider the cotangent bundle $\pi: T^{*} \mathbb{R}^{n} \longrightarrow \mathbb{R}^{n}$ over $\mathbb{R}^{n}$. Let $(x, p)=\left(x_{1}, \ldots, x_{n}, p_{1}, \ldots, p_{n}\right)$ be the canonical coordinate on $T^{*} \mathbb{R}^{n}$. Then the canonical symplectic structure on $T^{*} \mathbb{R}^{n}$ is given by the canonical two form $\omega=\sum_{i=1}^{n} d p_{i} \wedge d u_{i}$. Let $i: L \longrightarrow T^{*} \mathbb{R}^{n}$ be an immersion. We say that $i$ is a Lagrangian immersion if $\operatorname{dim} L=n$ and $i^{*} \omega=0$. In this case the critical value of $\pi \circ i$ is called the caustic of $i: L \longrightarrow T^{*} \mathbb{R}^{n}$ and it is denoted by $C_{L}$. The notion of Lagrangian submanifolds is a natural generalization of the notion of $C^{\infty}$-functions as follows: Let $F:\left(\mathbb{R}^{k} \times \mathbb{R}^{n},(0,0)\right) \longrightarrow(\mathbb{R}, 0)$ be an $n$-parameter unfolding of function germs. We call

$$
C(F)=\left\{(q, x) \in\left(\mathbb{R}^{k} \times \mathbb{R}^{n},(0,0)\right) \mid \frac{\partial F}{\partial q_{1}}(q, x)=\cdots=\frac{\partial F}{\partial q_{k}}(q, x)=0\right\},
$$

the catastrophe set of $F$ and

$$
\mathcal{B}_{F}=\left\{x \in\left(\mathbb{R}^{n}, 0\right) \mid \exists(q, x) \in C(F) \text { such that } \operatorname{rank}\left(\frac{\partial^{2} F}{\partial q_{i} \partial q_{j}}(q, x)\right)<k\right\}
$$

the bifurcation set of $F$. Let $\pi_{n}:\left(\mathbb{R}^{k} \times \mathbb{R}^{n}, 0\right) \longrightarrow\left(\mathbb{R}^{n}, 0\right)$ be the canonical projection, then we can easily show that the bifurcation set of $F$ is the critical value set of $\pi_{n} \mid C(F)$. We call $\pi_{C(F)}=\pi_{n} \mid C(F):(C(F), 0) \longrightarrow \mathbb{R}^{n}$ a catastrophe map of $F$. We say that $F$ is a Morse family of functions if the map germ

$$
\Delta F=\left(\frac{\partial F}{\partial q_{1}}, \ldots, \frac{\partial F}{\partial q_{k}}\right):\left(\mathbb{R}^{k} \times \mathbb{R}^{n}, 0\right) \longrightarrow\left(\mathbb{R}^{k}, 0\right)
$$

is non-singular, where $(q, x)=\left(q_{1}, \ldots, q_{k}, x_{1}, \ldots, x_{n}\right) \in\left(\mathbb{R}^{k} \times \mathbb{R}^{n}, 0\right)$. In this case we have a smooth submanifold germ $C(F) \subset\left(\mathbb{R}^{k} \times \mathbb{R}^{n}, 0\right)$ and a map germ $L(F):(C(F), 0) \longrightarrow T^{*} \mathbb{R}^{n}$ defined by

$$
L(F)(q, x)=\left(x, \frac{\partial F}{\partial x_{1}}, \ldots, \frac{\partial F}{\partial x_{n}}\right) .
$$

We can show that $L(F)$ is a Lagrangian immersion. Then we have the following fundamental theorem ([2], page 300).

Proposition 4.1 All Lagrangian submanifold germs in $T^{*} \mathbb{R}^{n}$ are constructed by the above method. 
Under the above notation, we call $F$ a generating family of $L(F)$. By definition, $C_{L(F)}=B_{F}$.

We define an equivalence relation among Lagrangian immersion germs. Let $i:(L, x) \longrightarrow$ $\left(T^{*} \mathbb{R}^{n}, p\right)$ and $i^{\prime}:\left(L^{\prime}, x^{\prime}\right) \longrightarrow\left(T^{*} \mathbb{R}^{n}, p^{\prime}\right)$ be Lagrangian immersion germs. Then we say that $i$ and $i^{\prime}$ are Lagrangian equivalent if there exist a diffeomorphism germ $\sigma:(L, x) \longrightarrow\left(L^{\prime}, x^{\prime}\right)$ , a symplectic diffeomorphism germ $\tau:\left(T^{*} \mathbb{R}^{n}, p\right) \longrightarrow\left(T^{*} \mathbb{R}^{n}, p^{\prime}\right)$ and a diffeomorphism germ $\bar{\tau}:\left(\mathbb{R}^{n}, \pi(p)\right) \longrightarrow\left(\mathbb{R}^{n}, \pi\left(p^{\prime}\right)\right)$ such that $\tau \circ i=i^{\prime} \circ \sigma$ and $\pi \circ \tau=\bar{\tau} \circ \pi$, where $\pi:\left(T^{*} \mathbb{R}^{n}, p\right) \longrightarrow$ $\left(\mathbb{R}^{n}, \pi(p)\right)$ is the canonical projection and a symplectic diffeomorphism germ is a diffeomorphism germ which preserves symplectic structure on $T^{*} \mathbb{R}^{n}$. In this case the caustic $C_{L}$ is diffeomorphic to the caustic $C_{L^{\prime}}$ by the diffeomorphism germ $\bar{\tau}$.

A Lagrangian immersion germ into $T^{*} \mathbb{R}^{n}$ at a point is said to be Lagrangian stable if for every map with the given germ there is a neighborhood in the space of Lagrangian immersions (in the Whitney $C^{\infty}$-topology) and a neighborhood of the original point such that each Lagrangian immersion belonging to the first neighborhood has in the second neighborhood a point at which its germ is Lagrangian equivalent to the original germ.

We can interpret the Lagrangian equivalence by using the notion of generating families.

Theorem 4.2 Let $F_{1} \in \mathfrak{M}_{k+n}$ and $F_{2} \in \mathfrak{M}_{k^{\prime}+n}$ be Morse families. Then we have the following: (1) $L\left(F_{1}\right)$ and $L\left(F_{2}\right)$ are Lagrangian equivalent if and only if $F_{1}, F_{2}$ are stably $P$ - $\mathcal{R}^{+}$-equivalent. (2) $L(F)$ is Lagrangian stable if and only if $F$ is an $\mathcal{R}^{+}$- versal deformation of $F \mid \mathbb{R}^{k} \times\{0\}$.

For the proof of the above theorem, see ([2], page 304 and 325).

By Theorem 2.3, the generating family of a generic Lagrangian stable map germ is stably $P$ - $\mathcal{R}^{+}$-equivalent to one of germs in the list of Theorem 2.3 for $n \leq 4$. Especially, we can draw the picture of caustics for $n=3$ :

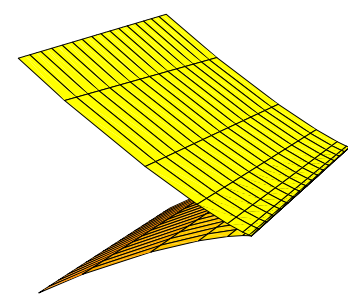

cuspidal edge

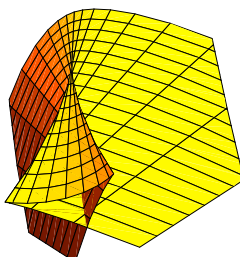

swallowtail

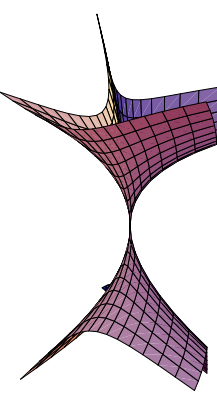

pyramid

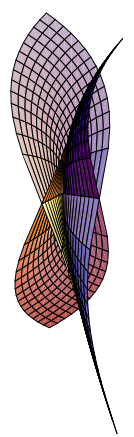

purse

Fig. 4.1

On the other hand, we now give a quick survey on the Legendrian singularity theory. Let $\pi$ : $P T^{*}\left(\mathbb{R}^{n}\right) \longrightarrow \mathbb{R}^{n}$ be the projective cotangent bundle over $\mathbb{R}^{n}$. This fibration can be considered as a Legendrian fibration with the canonical contact structure $K$ on $P T^{*}\left(\mathbb{R}^{n}\right)$. We now review geometric properties of this space. Consider the tangent bundle $\tau: T P T^{*}\left(\mathbb{R}^{n}\right) \rightarrow P T^{*}\left(\mathbb{R}^{n}\right)$ and the differential map $d \pi: T P T^{*}\left(\mathbb{R}^{n}\right) \rightarrow T \mathbb{R}^{n}$ of $\pi$. For any $X \in T P T^{*}\left(\mathbb{R}^{n}\right)$, there exists an element $\alpha \in T^{*}\left(\mathbb{R}^{n}\right)$ such that $\tau(X)=[\alpha]$. For an element $V \in T_{x}\left(\mathbb{R}^{n}\right)$, the property $\alpha(V)=0$ does not depend on the choice of representative of the class $[\alpha]$. Thus we can define the canonical contact structure on $P T^{*}\left(\mathbb{R}^{n}\right)$ by

$$
K=\left\{X \in T P T^{*}\left(\mathbb{R}^{n}\right) \mid \tau(X)(d \pi(X))=0\right\} .
$$


For the canonical coordinates $\left(x_{1}, \ldots, x_{n}\right)$ on $\mathbb{R}^{n}$, we have a trivialization $P T^{*}\left(\mathbb{R}^{n}\right) \cong \mathbb{R}^{n} \times$ $P\left(\mathbb{R}^{n-1}\right)^{*}$ and we call

$$
\left(\left(x_{1}, \ldots, x_{n}\right),\left[\xi_{1}: \cdots: \xi_{n}\right]\right)
$$

homogeneous coordinates, where $\left[\xi_{1}: \cdots: \xi_{n}\right]$ are homogeneous coordinates of the dual projective space $P\left(\mathbb{R}^{n-1}\right)^{*}$.

It is easy to show that $X \in K_{(x,[\xi])}$ if and only if $\sum_{i=1}^{n} \mu_{i} \xi_{i}=0$, where $d \tilde{\pi}(X)=\sum_{i=1}^{n} \mu_{i} \frac{\partial}{\partial x_{i}}$. An immersion $i: L \rightarrow P T^{*}\left(\mathbb{R}^{n}\right)$ is said to be a Legendrian immersion if $\operatorname{dim} L=n$ and $d i_{q}\left(T_{q} L\right) \subset K_{i(q)}$ for any $q \in L$. We also call the map $\pi \circ i$ the Legendrian map and the set $W(i)=$ image $\pi \circ i$ the wave front of $i$. Moreover, $i$ (or, the image of $i$ ) is called the Legendrian lift of $W(i)$.

The notion of Legendrian submanifolds is also a natural generalization of the notion of hypersurfaces. Let $F:\left(\mathbb{R}^{k} \times \mathbb{R}^{n}, 0\right) \longrightarrow(\mathbb{R}, 0)$ be a function germ. We say that $F$ is a Morse family of hypersurfaces if the mapping

$$
\Delta^{*} F=\left(F, \frac{\partial F}{\partial q_{1}}, \ldots, \frac{\partial F}{\partial q_{k}}\right):\left(\mathbb{R}^{k} \times \mathbb{R}^{n}, 0\right) \longrightarrow\left(\mathbb{R} \times \mathbb{R}^{k}, 0\right)
$$

is non-singular, where $(q, x)=\left(q_{1}, \ldots, q_{k}, x_{1}, \ldots, x_{n}\right) \in\left(\mathbb{R}^{k} \times \mathbb{R}^{n}, 0\right)$. In this case we have a smooth $(n-1)$-dimensional submanifold

$$
\Sigma_{*}(F)=\left\{(q, x) \in\left(\mathbb{R}^{k} \times \mathbb{R}^{n}, 0\right) \mid F(q, x)=\frac{\partial F}{\partial q_{1}}(q, x)=\cdots=\frac{\partial F}{\partial q_{k}}(q, x)=0\right\}
$$

and the map germ $\mathcal{L}(F):\left(\Sigma_{*}(F), 0\right) \longrightarrow P T^{*} \mathbb{R}^{n}$ defined by

$$
\mathcal{L}(F)(q, x)=\left(x,\left[\frac{\partial F}{\partial x_{1}}(q, x): \cdots: \frac{\partial F}{\partial x_{n}}(q, x)\right]\right)
$$

is a Legendrian immersion germ. Then we have the following fundamental theorem of Arnol'dZakalyukin

Proposition 4.3 All Legendrian submanifold germs in $P T^{*} \mathbb{R}^{n}$ are constructed by the above method.

We call $F$ a generating family of $\mathcal{L}(F)\left(\Sigma_{*}(F)\right)$. Therefore the wave front is

$$
W(\mathcal{L}(F))=\left\{x \in \mathbb{R}^{n} \mid \exists q \in \mathbb{R}^{k} \text { such that } F(q, x)=\frac{\partial F}{\partial q_{1}}(q, x)=\cdots=\frac{\partial F}{\partial q_{k}}(q, x)=0\right\} .
$$

We sometime write $\mathcal{D}_{F}=W(\mathcal{L}(F))$ and call it the discriminant set of $F$.

We now introduce an equivalence relation among Legendrian immersion germs. Let $i$ : $(L, p) \subset\left(P T^{*} \mathbb{R}^{n}, p\right)$ and $i^{\prime}:\left(L^{\prime}, p^{\prime}\right) \subset\left(P T^{*} \mathbb{R}^{n}, p^{\prime}\right)$ be Legendrian immersion germs. Then we say that $i$ and $i^{\prime}$ are Legendrian equivalent if there exists a contact diffeomorphism germ $H:\left(P T^{*} \mathbb{R}^{n}, p\right) \longrightarrow\left(P T^{*} \mathbb{R}^{n}, p^{\prime}\right)$ such that $H$ preserves fibers of $\pi$ and that $H(L)=L^{\prime}$. A Legendrian immersion germ $i:(L . p) \subset P T^{*} \mathbb{R}^{n}$ (or, a Legendrian map $\pi \circ i$ ) at a point is said to be Legendrian stable if for every map with the given germ there is a neighborhood in the space of Legendrian immersions (in the Whitney $C^{\infty}$ topology) and a neighborhood of the original point such that each Legendrian immersion belonging to the first neighborhood has in the second neighborhood a point at which its germ is Legendrian equivalent to the original germ. Since the Legendrian lift $i:(L, p) \subset\left(P T^{*} \mathbb{R}^{n}, p\right)$ is uniquely determined on the regular part of the wave front $W(i)$, we have the following simple but significant property of Legendrian immersion germs: 
Proposition 4.4 Let $i:(L, p) \subset\left(P T^{*} \mathbb{R}^{n}, p\right)$ and $i^{\prime}:\left(L^{\prime}, p^{\prime}\right) \subset\left(P T^{*} \mathbb{R}^{n}, p^{\prime}\right)$ be Legendrian immersion germs such that regular sets of $\pi \circ i, \pi \circ i^{\prime}$ are dense respectively. Then $i, i^{\prime}$ are Legendrian equivalent if and only if wave front sets $W(i), W\left(i^{\prime}\right)$ are diffeomorphic as set germs.

This result has been firstly pointed out by Zakalyukin [80]. The assumption in the above proposition is a generic condition for $i, i^{\prime}$. Specially, if $i, i^{\prime}$ are Legendrian stable, then these satisfy the assumption.

We can interpret the Legendrian equivalence by using the notion of generating families. Let $F, G:\left(\mathbb{R}^{k} \times \mathbb{R}^{n}, 0\right) \longrightarrow(\mathbb{R}, 0)$ be $n$-parameter unfoldings of function germs. We say that $F$ and $G$ are $P$-K $\mathcal{K}$-equivalent if there exists a diffeomorphism germ $\Psi:\left(\mathbb{R}^{k} \times \mathbb{R}^{n}, 0\right) \longrightarrow\left(\mathbb{R}^{k} \times \mathbb{R}^{n}, 0\right)$ of the form $\Psi(x, u)=\left(\psi_{1}(q, x), \psi_{2}(x)\right)$ for $(q, x) \in\left(\mathbb{R}^{k} \times \mathbb{R}^{n}, 0\right)$ such that $\Psi^{*}\left(\langle F\rangle_{\mathcal{E}_{k+n}}\right)=\langle G\rangle_{\mathcal{E}_{k+n}}$. Here $\Psi^{*}: \mathcal{E}_{k+n} \longrightarrow \mathcal{E}_{k+n}$ is the pull back $\mathbb{R}$-algebra isomorphism defined by $\Psi^{*}(h)=h \circ \Psi$. If $n=0$, we simply say these germs are $\mathcal{K}$-equivalent.

Let $F:\left(\mathbb{R}^{k} \times \mathbb{R}^{3}, 0\right) \longrightarrow(\mathbb{R}, 0)$ be a function germ. We say that $F$ is a $\mathcal{K}$-versal deformation of $f=F \mid \mathbb{R}^{k} \times\{0\}$ if

$$
\mathcal{E}_{k}=T_{e}(\mathcal{K})(f)+\left\langle\frac{\partial F}{\partial x_{1}}\left|\mathbb{R}^{k} \times\{0\}, \ldots, \frac{\partial F}{\partial x_{n}}\right| \mathbb{R}^{k} \times\{0\}\right\rangle_{\mathbb{R}},
$$

where

$$
T_{e}(\mathcal{K})(f)=\left\langle\frac{\partial f}{\partial q_{1}}, \ldots, \frac{\partial f}{\partial q_{k}}, f\right\rangle_{\mathcal{E}_{k}}
$$

(See [48].)

The main result in Arnol'd-Zakalyukin's theory [2, 79] is the following:

Proposition 4.5 Let $F, G:\left(\mathbb{R}^{k} \times \mathbb{R}^{n}, 0\right) \longrightarrow(\mathbb{R}, 0)$ be Morse families of hypersurfaces. Then (1) $\mathcal{L}(F)$ and $\mathcal{L}(G)$ are Legendrian equivalent if and only if $F, G$ are $P$-K $\mathcal{K}$-equivalent.

(2) $\mathcal{L}(F)$ is Legendrian stable if and only if $F$ is a $\mathcal{K}$-versal deformation of $F \mid \mathbb{R}^{k} \times\{0\}$.

Since $F, G$ are function germs on the common space germ $\left(\mathbb{R}^{k} \times \mathbb{R}^{n}, 0\right)$, we do no need the notion of stably $P$ - $\mathcal{K}$-equivalences under this situation (cf., [2]). By the uniqueness result of the $\mathcal{K}$-versal deformation of a function germ, Propositions 4.5 and 4.6 , we have the following classification result of Legendrian stable germs. For any map germ $f:\left(\mathbb{R}^{n}, 0\right) \longrightarrow\left(\mathbb{R}^{p}, 0\right)$, we define the local ring of $f$ by $Q(f)=\mathcal{E}_{n} / f^{*}\left(\mathfrak{M}_{p}\right) \mathcal{E}_{n}$.

Proposition 4.6 Let $F, G:\left(\mathbb{R}^{k} \times \mathbb{R}^{n}, 0\right) \longrightarrow(\mathbb{R}, 0)$ be Morse families. Suppose that $\mathcal{L}(F), \mathcal{L}(G)$ are Legendrian stable. The the following conditions are equivalent.

(1) $(W(\mathcal{L}(F)), 0)$ and $(W(\mathcal{L}(G)), 0)$ are diffeomorphic as germs.

(2) $\mathcal{L}(F)$ and $\mathcal{L}(G)$ are Legendrian equivalent.

(3) $Q(f)$ and $Q(g)$ are isomorphic as $\mathbb{R}$-algebras, where $f=F\left|\mathbb{R}^{k} \times\{0\}, g=G\right| \mathbb{R}^{k} \times\{0\}$.

We also have the following classification of $\mathcal{K}$-versal deformation under the $P$ - $\mathcal{K}$-equivalence.

Theorem 4.7 Let $f:\left(\mathbb{R}^{k}, 0\right) \longrightarrow(\mathbb{R}, 0)$ be a smooth function germ with

$$
2 \leq \tau(f)=\operatorname{dim}_{\mathbb{R}} \frac{\mathcal{E}_{k}}{T_{e}(\mathcal{K}(f)} \leq 4 .
$$

The $\tau(f)$-parameter $\mathcal{R}^{+}$-versal unfolding of $f$ is stably $P-\mathcal{R}^{+}$-equivalent to one of the following unfoldings: 
(1) $q_{1}^{3}+x_{1} q_{1}+x_{2}$

(2) $q_{1}^{4}+x_{1} q_{1}^{2}+x_{2} q_{1}+x_{3}$

(3) $q_{1}^{5}+x_{1} q_{1}^{3}+x_{2} q_{1}^{2}+x_{3} q_{1}+x_{4}$,

(4) $q_{1}^{3}+q_{2}^{3}+x_{1} q_{1} q_{2}+x_{2} q_{1}+x_{3} q_{2}+x_{4}$,

(5) $q_{1}^{3}-q_{1} q_{2}+x_{1}\left(q_{1}^{2}+q_{2}^{2}\right)+x_{2} q_{1}+x_{3} q_{2}+x_{4}$.

By the above theorem, the wave front of a stable Legendrian submanifold in $\mathbb{R}^{3}$ is the cuspidaledge or the swallowtail.

\section{Solid shapes and differential geometry}

We might say that the classical mathematical theory on the "solid shape" is differential geometry of curves and surfaces. It is deeply related to the vision theory and the computer graphics. Porteous is the first person who pointed out the importance of "landmarks" or "robust futures" of curves and surfaces. These are futures of the curve or the surface that preserve some sort of individual identity if the curve or the surface is subjected to small deformation. Familiar examples are inflection points or vertices of curves and parabolic points or umbilic points of surfaces. Less familiar are ridges which were discovered by Porteous[65].

We now start to consider landmarks of plane curves. Let $\gamma: I \longrightarrow \mathbb{R}^{2}$ be a unit speed plane curve (i.e., $\left\|\boldsymbol{\gamma}^{\prime}(s)\right\|=1$, where $\boldsymbol{\gamma}^{\prime}(s)=(d \boldsymbol{\gamma} / d s)(s)$ ). Let us denote that $\boldsymbol{t}(s)=\boldsymbol{\gamma}^{\prime}(s)$ and we call $\boldsymbol{t}(s)$ a unit tangent vector of $\boldsymbol{\gamma}$ at $s$. We define a unit vector $\boldsymbol{n}(s)$ which is given by the $\pi / 2$-rotation of $\boldsymbol{t}(s)$ to anti-clockwise direction and we call $\boldsymbol{n}(s)$ a unit normal vector of $\boldsymbol{\gamma}$ at $s$. The we have the following Frenet formulae:

$$
\left\{\begin{aligned}
\boldsymbol{t}^{\prime}(s) & =\kappa(s) \boldsymbol{n}(s) \\
\boldsymbol{n}^{\prime}(s) & =-\kappa(s) \boldsymbol{t}(s)
\end{aligned}\right.
$$

where $\kappa(s)=x_{1}^{\prime}(s) x_{2}^{\prime \prime}(s)-x_{1}^{\prime \prime}(s) x_{2}^{\prime}(s)$ is called the curvature of $\gamma$ at $s$. The inflection point of $\gamma$ is a point $\gamma(s)$ with $\kappa(s)=0$ and the vertex of $\boldsymbol{\gamma}$ is a point $\gamma(s)$ with $\kappa^{\prime}(s)=0$. Why can we say such the points are the landmarks of $\gamma$ ? Since the curve is smooth, we cannot distinguish such the points from the view point of differential topology. For example we can not easily discern between two curves in the Fig.5.1. The picture (a) is a circle $\gamma(s)=(\cos s, \sin s)$ and the picture (b) is a ellipse $\gamma(s)=(1.01 \cos s, \sin s)$.

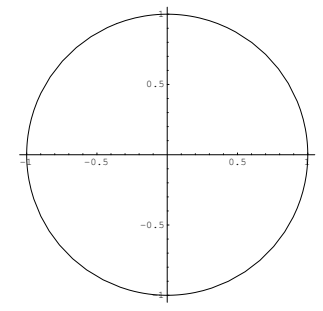

(a) circle

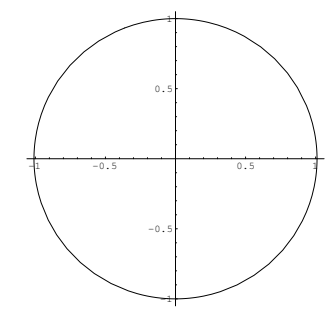

(b) ellipse

Fig. 5.1

On the other hand, we now consider the following new curves associated to $\gamma$ :

$$
\begin{aligned}
E V_{\gamma}(s) & =\gamma(s)+\frac{1}{\kappa(s)} \boldsymbol{n}(s),(\kappa(s) \neq 0) \\
P e_{\gamma}(s) & =(\gamma(s) \cdot \boldsymbol{n}(s)) \boldsymbol{n}(s),
\end{aligned}
$$


where $\boldsymbol{a} \cdot \boldsymbol{b}$ denotes the canonical scalar product in $\mathbb{R}^{2}$. We respectively call $E V_{\gamma}$ the evolute and $P e_{\gamma}$ the pedal of $\gamma$. By the Frenet formulae, we can show that

$$
\begin{aligned}
E V_{\gamma}^{\prime}(s)= & -\frac{\kappa^{\prime}(s)}{\kappa^{2}(s)} \boldsymbol{n}(s) \\
P e_{\gamma}^{\prime}(s)= & -\kappa(s)\{(\boldsymbol{\gamma}(s) \cdot \boldsymbol{t}(s)) \boldsymbol{n}(s) \\
& +(\gamma(s) \cdot \boldsymbol{n}(s)) \boldsymbol{t}(s)\}
\end{aligned}
$$

so that $E V_{\gamma}^{\prime}\left(s_{0}\right)=0$ (respectively , $P e_{\gamma}^{\prime}\left(s_{0}\right)=0$ ) if and only if $\kappa^{\prime}\left(s_{0}\right)=0$ (respectively, $\left.\kappa\left(s_{0}\right)=0\right)$. This means that the singularities of $E V_{\gamma}$ (respectively, $P e_{\gamma}$ ) correspond to the vertices (respectively, inflections). In fact, we can draw the evolute of an ellipse in Fig. 5.2.

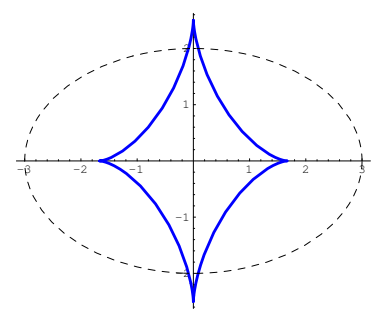

Fig. 5.2 : The dotted line is the ellipse and the solid line is the evolute.

Moreover we also draw the pedal of lemniscate in Fig 5.3. Moreover, if we draw the picture of

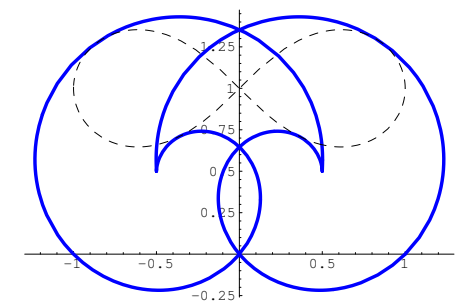

Fig. 5.3 : The dotted line is the lemniscate and the solid line is the pedal.

the evolute corresponding to the ellipse in Fig. 5.1, we can recognize the four cusp singularities (cf., Fig. 5.4). Since the landmarks have robust future, it is very useful in the vision theory and

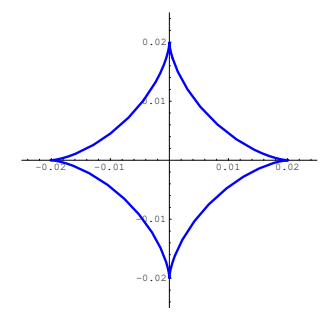

Fig. 5.4 : The evolute of the ellipse of Fig. 5.1, (b).

the computer graphics. We can study the singularities of evolutes and pedals as applications of the theory of singularities of smooth functions.

We now define a family of functions

$$
D: I \times \mathbb{R}^{2} \longrightarrow \mathbb{R}
$$


by $D(s, \boldsymbol{x})=\|\boldsymbol{\gamma}(s)-\boldsymbol{x}\|^{2}$ which we call the distance squared function of $\boldsymbol{\gamma}$. We also define a function

$$
H: I \times\left(\mathbb{R}^{2} \backslash\{0\}\right) \longrightarrow \mathbb{R}
$$

by $H(s, \boldsymbol{v})=\gamma(s) \cdot(\boldsymbol{v} /\|\boldsymbol{v}\|)-\|\boldsymbol{v}\|$, where $\|\boldsymbol{v}\|$ is the norm of $\boldsymbol{v}$. We call $H$ the extended height function of $\boldsymbol{\gamma}$. We define that $d_{x}(s)=D(s, \boldsymbol{x})$ and $h_{v}(s)=H(s, \boldsymbol{v})$, then we have the following proposition

Proposition 5.1 Let $\gamma: I \longrightarrow \mathbb{R}^{2}$ be a unit speed plane curve. Then we have the following:

(A) $d_{x}^{\prime}(s)=d_{x}^{\prime \prime}(s)=0$ if and only if $\kappa(s) \neq 0$ and $\boldsymbol{x}=\gamma(s)+\frac{1}{\kappa(s)} \boldsymbol{n}(s)$.

(B) $h_{v}(s)=h_{v}^{\prime}(s)=0$ if and only if $\boldsymbol{v}=(\gamma(s) \cdot \boldsymbol{n}(s)) \boldsymbol{n}(s)$.

Therefore, the bifurcation set $\mathcal{B}_{D}$ is the evolute and the discriminant set $\mathcal{D}_{H}$ is the pedal of $\boldsymbol{\gamma}$. This means that the evolute of $\gamma$ is a caustic and the pedal of $\gamma$ is a wave front. We know that generic caustics and wave fronts on a plane are non-singular curves or 3/2-cusps as an application of the classification theorem in $\S 4$. It is the reason why we can always observe cusps on the evolute and the pedal in Figures 5.2-5.4.

From the view point of the vision theory, surfaces in $\mathbb{R}^{3}$ are important much more than plane curves. We now briefly review the classical extrinsic differential geometry of surfaces. Let $\boldsymbol{X}: U \rightarrow \mathbb{R}^{3}$ be an embedding, where $U \subset \mathbb{R}^{2}$ is an open subset. We denote that $M=\boldsymbol{X}(U)$ and identify $M$ and $U$ through the embedding $\boldsymbol{X}$. The tangent space of $M$ at $p=\boldsymbol{X}(u)$ is

$$
T_{p} M=\left\langle\boldsymbol{X}_{u_{1}}(u), \boldsymbol{X}_{u_{2}}(u)\right\rangle_{\mathbb{R}}
$$

We can define the unit normal vector field

$$
\boldsymbol{n}(u)=\frac{\boldsymbol{X}_{u_{1}}(u) \times \boldsymbol{X}_{u_{2}}(u)}{\left\|\boldsymbol{X}_{u_{1}}(u) \times \boldsymbol{X}_{u_{2}}(u)\right\|}
$$

along $\boldsymbol{X}: U \longrightarrow \mathbb{R}^{3}$, where $\boldsymbol{a} \times \boldsymbol{b}$ is the vector product of $\boldsymbol{a}, \boldsymbol{b}$. A map $G: U \longrightarrow S^{2}$ defined by $G(u)=\boldsymbol{n}(u)$ is called the Gauss map of $M=\boldsymbol{X}(U)$. We can easily show that $D_{v} \boldsymbol{n} \in T_{p} M$ for any $p=\boldsymbol{X}(u) \in M$ and $\boldsymbol{v} \in T_{p} M$. Here $D_{v}$ denotes the covariant derivative with respect to the tangent vector $\boldsymbol{v}$. Therefore the derivative of the Gauss map $d G(u)$ can be interpreted as a liner transformation on the tangent space $T_{p} M$ at $p=\boldsymbol{X}(u)$. We call the linear transformation $S_{p}=-d G(u): T_{p} M \longrightarrow T_{p} M$ the shape operator (or Weingarten map) of $M=\boldsymbol{X}(U)$ at $p=\boldsymbol{X}(u)$. We denote the eigenvalues of $S_{p}$ by $\kappa_{i}(p)(i=1,2)$ which we call a principal curvature. We call the eigenvector of $S_{p}$ the principal direction. By definition, $\kappa_{p}$ is a principal curvature if and only if $\operatorname{det}\left(S_{p}-\kappa_{p} I\right)=0$. The Gauss curvature of $M=\boldsymbol{X}(U)$ at $p=\boldsymbol{X}(u)$ is defined to be $K(u)=\operatorname{det} S_{p}$.

We say that a point $p=\boldsymbol{X}(u) \in M$ is an umbilic point if $S_{p}=k(p) 1_{T_{p} M}$ (i.e., $\kappa_{1}(p)=\kappa_{2}(p)$ ). We also say that $M$ is totally umbilic if all points of $M$ are umbilic. Then the following proposition is a well-known result:

Proposition 5.2 Suppose that $M=\boldsymbol{X}(U)$ is totally umbilic, then $\kappa(p)$ is constant $\kappa$. Under this condition, we have the following classification:

1) If $\kappa \neq 0$, then $M$ is a part of a sphere.

2) If $\kappa=0$, then $M$ is a part of a plane. 
In the extrinsic differential geometry, totally umbilic surfaces are considered to be the model surfaces in Euclidean space. Since $\left\{\boldsymbol{X}_{u_{1}}, \boldsymbol{X}_{u_{2}}\right\}$ is linearly independent, we induce the Riemannian metric (first fundamental form) $d s^{2}=\sum_{i=1}^{2} g_{i j} d u_{i} d u_{j}$ on $M=\boldsymbol{X}(U)$, where $g_{i j}(u)=\left\langle\boldsymbol{X}_{u_{i}}(u), \boldsymbol{X}_{u_{j}}(u)\right\rangle$ for any $u \in U$. We define the second fundamental invariant by $h_{i j}(u)=\left\langle-\boldsymbol{n}_{u_{i}}(u), \boldsymbol{X}_{u_{j}}(u)\right\rangle$ for any $u \in U$. We have the following Weingarten formula:

$$
\boldsymbol{n}_{u_{i}}(u)=-\sum_{j=1}^{2} h_{i}^{j}(u) \boldsymbol{X}_{u_{j}}(u)
$$

where $\left(h_{i}^{j}(u)\right)=\left(h_{i k}(u)\right)\left(g^{k j}(u)\right)$ and $\left(g^{k j}(u)\right)=\left(g_{k j}(u)\right)^{-1}$. By the Weingarten formula, the Gauss curvature is given by

$$
K(u)=\frac{\operatorname{det}\left(h_{i j}(u)\right)}{\operatorname{det}\left(g_{\alpha \beta}(u)\right)} .
$$

For a surface $\boldsymbol{X}: U \longrightarrow \mathbb{R}^{3}$, we say that a point $u \in U$ or $p=\boldsymbol{X}(u)$ is a flat point if $h_{i j}(u)=0$ for all $i, j$. Therefore, $p=\boldsymbol{X}(u)$ is a flat point if and only if $p$ is an umbilic point with the vanishing principal curvature. We say that a point $p=\boldsymbol{X}(u) \in M$ is a parabolic point if $K(u)=0$. For a surface $\boldsymbol{X}: U \longrightarrow \mathbb{R}^{3}$, we define the evolute of $\boldsymbol{X}(U)=M$ by

$$
\operatorname{Ev}_{M}=\left\{\boldsymbol{X}(u)+\frac{1}{\kappa(u)} \boldsymbol{n}(u) \mid \kappa(u) \text { is a principal curvature at } p=\boldsymbol{X}(u), u \in U\right\} .
$$

The evolute is also called the focal set of $M$. We define a smooth mapping $\operatorname{Ev}_{\kappa}: U \longrightarrow \mathbb{R}^{3}$ by

$$
\operatorname{Ev}_{\kappa}(u)=\boldsymbol{X}(u)+\frac{1}{\kappa(u)} \boldsymbol{n}(u)
$$

where we fix a principal curvature $\kappa(u)$ on $U$ at $u$ with $\kappa(u) \neq 0$. This map gives a parametrization of a component of $\mathrm{Ev}_{M}$. We also define the pedal surface of $M=\boldsymbol{X}(U)$ by

$$
\mathrm{Pe}_{M}: U \longrightarrow \mathbb{R}^{n} ; \mathrm{Pe}_{M}(u)=\langle\boldsymbol{X}(u), \boldsymbol{n}(u)\rangle \boldsymbol{n}(u) .
$$

Concerning on the pedal surface in $\mathbb{R}^{3}$, we define the cylindrical pedal of $M=\boldsymbol{X}(U)$ by

$$
\mathrm{CPe}_{M}: U \longrightarrow S^{2} \times \mathbb{R} ; \mathrm{CPe}_{M}(u)=(\boldsymbol{n}(u),\langle\boldsymbol{X}(u), \boldsymbol{n}(u)\rangle) .
$$

The cylindrical pedal of $M$ is called the dual of $M$ in $[9,67]$. We have the following well-known result:

Proposition 5.3 Let $M=\boldsymbol{X}(U)$ be a surface in $\mathbb{R}^{3}$.

(a) Suppose that there are no parabolic points or flat points, then the following are equivalent:

(1) $M$ is totally umbilic with $\kappa \neq 0$.

(2) $\mathrm{Ev}_{M}$ is a point in $\mathbb{R}^{3}$.

(3) $M$ is a part of a sphere.

(b) The following are equivalent:

(1) $M$ is totally umbilic with $\kappa=0$.

(2) The Gauss map is a constant map.

(3) $M$ is a part of a plane. 
We define a mapping $\Psi: S^{2} \times(\mathbb{R} \backslash\{0\}) \longrightarrow \mathbb{R}^{3} \backslash\{0\}$ by $\Psi(\boldsymbol{v}, r)=r \boldsymbol{v}$. We can easily show that $\Psi$ is a double covering and $\Psi\left(\mathrm{CPe}_{M}(u)\right)=\mathrm{Pe}_{M}(u)$ under the assumption that $\langle\boldsymbol{X}(u), \boldsymbol{n}(u)\rangle \neq 0$. If necessary, by applying a Euclidean motion in $\mathbb{R}^{3}$, we have the condition $\langle\boldsymbol{X}(u), \boldsymbol{n}(u)\rangle \neq 0$. Since we consider the geometric properties which are invariant under Euclidean motion, we might assume the above condition. Therefore the singularities of the pedal and the cylindrical pedal of a hypersurface are diffeomorphic. Although the notion of pedals are classically given, we consider the cylindrical pedal instead of the pedal of $M=\boldsymbol{X}(U)$ by the above reason.

We now define two families of functions

$$
H: U \times S^{2} \longrightarrow \mathbb{R}
$$

by $H(u, \boldsymbol{v})=\langle\boldsymbol{X}(u), \boldsymbol{v}\rangle$ and

$$
D: U \times \mathbb{R}^{3} \longrightarrow \mathbb{R}
$$

by $D(u, \boldsymbol{x})=\|\boldsymbol{X}(u)-\boldsymbol{x}\|^{2}$. We call $H$ a height function and $D$ a distance squared function) on $M=\boldsymbol{X}(U)$. We denote that $h_{v}(u)=H(u, \boldsymbol{v})$ and $d_{x}(u)=D(u, \boldsymbol{x})$. These two families of functions are introduced by Thom for the study of parabolic points and umbilical points. Actually, Porteous and Montaldi realized Thom's program $[57,65,66]$. The following proposition follows from direct calculations:

Proposition 5.4 Let $\boldsymbol{X}: U \longrightarrow \mathbb{R}^{n}$ be a hypersurface. Then

(1) $\left(\partial h_{v} / \partial u_{i}\right)(u)=0(i=1, \ldots, n-1)$ if and only if $\boldsymbol{v}= \pm \boldsymbol{n}(u)$.

(2) $\left(\partial d_{x} / \partial u_{i}\right)(u)=0(i=1, \ldots, n-1)$ if and only if there exist real numbers $\lambda$ such that $\boldsymbol{v}=\boldsymbol{x}(u)+\lambda \boldsymbol{n}(u)$.

By Proposition 5.4, we can detect both the catastrophe sets of $H$ and $D$ as follows:

$$
\begin{gathered}
C(H)=\left\{(u, \boldsymbol{v}) \in U \times S^{2} \mid \boldsymbol{v}= \pm \boldsymbol{n}(u)\right\}, \\
C(D)=\left\{(u, \boldsymbol{x}) \in U \times \mathbb{R}^{3} \mid \boldsymbol{x}=\boldsymbol{x}(u)+\mu \boldsymbol{n}(u)\right\} .
\end{gathered}
$$

For $\boldsymbol{v}=\boldsymbol{n}(u)$, We also calculate that

$$
\frac{\partial^{2} H}{\partial u_{i} \partial u_{j}}(u, \boldsymbol{v})=\left\langle\boldsymbol{X}_{u_{i} u_{j}}(u), \boldsymbol{v}\right\rangle=\mp h_{i j}(u)
$$

on $C(H)$ and

$$
\frac{\partial^{2} D}{\partial u_{i} \partial u_{j}}(u, \boldsymbol{x})=2\left(\left\langle\boldsymbol{X}_{u_{i} u_{j}}(u), \boldsymbol{X}(u)-\boldsymbol{x}\right\rangle+\left\langle\boldsymbol{X}_{u_{j}}(u), \boldsymbol{X}_{u_{j}}(u)\right\rangle\right)=2\left(-\lambda h_{i j}(u)+g_{i j}(u)\right)
$$

on $C(D)$.

Therefore, for any $\left.\boldsymbol{v}=\boldsymbol{n}(u), \operatorname{det}\left(\mathcal{H}\left(h_{v}\right)(u)\right)=\operatorname{det}\left(\partial^{2} H / \partial u_{i} \partial u_{j}\right)(u, \boldsymbol{v})\right)=0$ if and only if $K(p)=0$ (i.e., $p=\boldsymbol{X}(u)$ is a parabolic point). Moreover, for any $\boldsymbol{x}=\boldsymbol{X}(u)+\lambda \boldsymbol{n}(u)$, $\left.\operatorname{det}\left(\mathcal{H}\left(d_{x}\right)(u)\right)=\operatorname{det}\left(\partial^{2} D / \partial u_{i} \partial u_{j}\right)(u, \boldsymbol{x})\right)=0$ if and only if $\kappa(u)=\frac{1}{\lambda}$ is a principal curvature. By the above calculation, we have the following well-known results:

Proposition 5.5 For any $p=\boldsymbol{X}(u)$, we have the following assertions:

Suppose that $\boldsymbol{v}=\boldsymbol{n}(u)$, then 
(a) $p$ is a parabolic point if and only if $\operatorname{det}\left(\mathcal{H}\left(h_{v}\right)(u)\right)=0$.

(b) $p$ is a flat point if and only if $\operatorname{rank} \mathcal{H}\left(h_{v}\right)(u)=0$.

Suppose that $p$ is not a flat point and $\boldsymbol{x}=\boldsymbol{X}(u)+(1 / \kappa(u)) \boldsymbol{n}(u)$ for a non-zero principal curvature $\kappa(u)$. Then

(c) $p$ is an umbilical point if and only if $\operatorname{rank} \mathcal{H}\left(d_{x}\right)(u)=0$.

We say that $u$ is a ridge point if $h_{v}$ has the $A_{k \geq 3}$-type singular point at $u$, where $\boldsymbol{v} \in \operatorname{Ev}_{M}(U)$. For a function germ $f:\left(\mathbb{R}^{n}, \boldsymbol{x}_{0}\right) \longrightarrow \mathbb{R}, f$ has $A_{k}$-type singular point at $\boldsymbol{x}_{0}$ if $f$ is $\mathcal{R}^{+}$-equivalent to the germ $x_{1}^{k+1} \pm x_{2}^{2} \pm \cdots \pm x_{n}^{2}$. We say that two function germs $f_{i}:\left(\mathbb{R}^{n}, \boldsymbol{x}_{i}\right) \longrightarrow \mathbb{R}(i=1,2)$ are $\mathcal{R}^{+}$-equivalent if there exists a diffeomorphism germ $\Phi:\left(\mathbb{R}^{n}, \boldsymbol{x}_{1}\right) \longrightarrow\left(\mathbb{R}^{n}, \boldsymbol{x}_{2}\right)$ and a real number $c$ such that $f_{2} \circ \Phi(\boldsymbol{x})=f_{2}(\boldsymbol{x})+c$. The notion of ridge points was introduced by Porteous[65] as an application of the singularity theory of unfoldings to the evolute and the geometric meaning of ridge points is given as follows: Let $F: \mathbb{R}^{3} \longrightarrow \mathbb{R}$ be a function and $\boldsymbol{X}: U \longrightarrow \mathbb{R}^{3}$ a surface. We say that $\boldsymbol{X}$ and $F^{-1}(0)$ have a corank $r$ contact at $p=\boldsymbol{X}(u)$ if the Hessian of the function $g(u)=F \circ \boldsymbol{X}(u)$ has corank $r$ at $u$. We also say that $\boldsymbol{X}$ and $F^{-1}(0)$ have an $A_{k}$-type contact at $p=\boldsymbol{X}(u)$ if the function $g(u)=F \circ \boldsymbol{X}(u)$ has the $A_{k}$-type singularity at $u$. By definition, if $\boldsymbol{X}$ and $F^{-1}(0)$ have an $A_{k}$-type contact at $p=\boldsymbol{X}(u)$, then these have a corank 1 contact. For any $r \in \mathbb{R}$ and $\boldsymbol{a}_{0} \in \mathbb{R}^{3}$, we consider a function $F: \mathbb{R}^{3} \longrightarrow \mathbb{R}$ defined by $F(\boldsymbol{x})=\left\|\boldsymbol{x}-\boldsymbol{a}_{0}\right\|^{2}-r^{2}$. We denote that

$$
S^{2}(\boldsymbol{a}, r)=F^{-1}(0)=\left\{\boldsymbol{u} \in \mathbb{R}^{3} \mid\|\boldsymbol{x}-\boldsymbol{a}\|^{2}=r^{2}\right\} .
$$

It follows that $S^{2}(\boldsymbol{a}, r)$ is a sphere with the center $\boldsymbol{a}$ and the radius $|r|$. We put $\boldsymbol{a}=\operatorname{Ev}_{\kappa}(u)$ and $r=1 / \kappa(u)$, where we fix a principal curvature $\kappa(u)$ on $U$ at $u$, then we have the following simple proposition:

Proposition 5.6 Under the above notations, there exists an integer $\ell=1$ or 2 such that $M=\boldsymbol{X}(U)$ and $S^{2}(\boldsymbol{a}, r)$ have corank $\ell$ contact at $u$.

In the above proposition, $S^{2}(\boldsymbol{a}, r)$ is called a focal sphere of $M=\boldsymbol{X}(U)$. We also call $\boldsymbol{a}$ the focal center $\kappa(u)$. By Proposition 5.6, $M=\boldsymbol{X}(U)$ and the focal sphere has corank 2 contact at an umbilic point. Therefore the ridge point is not an umbilic point. We also consider another geometric meaning of ridge points. A curve $\gamma(t)=\boldsymbol{X}(u(t), v(t))$ on a surface $M=\boldsymbol{X}(U)$ is a line of curvature if the tangent vector $\dot{\gamma}(t)$ is a principal direction for any $t$. We have the following proposition $[65,66]$.

Proposition 5.7 Let $\boldsymbol{\gamma}(t)=\boldsymbol{X}(u(t), v(t))$ be a line of curvature on a surface $M=\boldsymbol{X}(U)$ and $\kappa(t)$ the principal curvature with respect to the principal direction $\dot{\gamma}(t)$. Then $p=\boldsymbol{X}\left(u\left(t_{0}\right), v\left(t_{0}\right)\right)$ is a ridge point if and only if $\dot{\kappa}\left(t_{0}\right)=0$.

Porteous discovered the notion of ridge points when he investigated the singular points of the evolute $\operatorname{Ev}_{M}[65]$.

By the general theory of unfoldings of function germs, the bifurcation set $\mathcal{B}_{F}$ is non-singular at the origin if and only if the function $f=F \mid \mathbb{R}^{n} \times\{0\}$ has the $A_{2}$-type singularity (i.e., the fold type singularity). Therefore we have the following proposition:

Proposition 5.8 Under the same notations as in the previous proposition, the evolute $\mathrm{Ev}_{M}$ is non-singular at $\boldsymbol{a}=\mathrm{Ev}_{\kappa}(u)$ if and only if $M=\boldsymbol{X}(U)$ and $S^{2}(\boldsymbol{a}, r)$ have $A_{2}$-type contact at $u$. 
All results mentioned in the above paragraphs on the evolute have been shown by Porteous and Montaldi[57, 65].

We also define a family of functions $\widetilde{H}: U \times\left(S^{2} \times \mathbb{R}\right) \longrightarrow \mathbb{R}$ by

$$
\widetilde{H}(u, \boldsymbol{v}, r)=\langle\boldsymbol{X}(u), \boldsymbol{v}\rangle-r .
$$

We call it the extended height function of $M=\boldsymbol{X}(U)$. By the previous calculations, we have

$$
\mathcal{D}_{\widetilde{H}}=\left\{ \pm \operatorname{CPe}_{M}(u) \mid u \in U\right\} \quad \text { and } \quad \mathcal{B}_{D}=\operatorname{Ev}_{M} .
$$

Moreover, the catastrophe map of $H$ is $\pi_{C(H)}(u, \pm \boldsymbol{n}(u))= \pm \boldsymbol{n}(u)= \pm G(u)$. Therefore, we can identify the Gauss map of $M=\boldsymbol{X}(U)$ with the positive component of the catastrophe map $\pi_{C(H)}$.

For a surface $\boldsymbol{X}: U \longrightarrow \mathbb{R}^{3}$, we consider the distance squared function $D$ and the height function $H$. We have the following propositions(cf., [39]):

Proposition 5.9 Both of the distance squared function $D: U \times \mathbb{R}^{n} \longrightarrow \mathbb{R}$ and the height function $H: U \times S^{2} \longrightarrow \mathbb{R}$ of $M=\boldsymbol{X}(U)$ are Morse families of functions. Moreover, the extended height function of $M=\boldsymbol{X}(U)$ is a Morse family of hypersurfaces.

By this proposition, the evolute is a caustics and the pedal is a wave front. Therefore, we can apply the theory of Lagrangian or Legendrian singularities to the study of the landmarks of surfaces[39].

On the other hand, we now consider the image of orthogonal projection of a surface in $\mathbb{R}^{3}$. For orthogonal projection in the unit direction $\boldsymbol{k}$ we can take an image plane through the origin 0 , that is an image plane with equation $\boldsymbol{x} \cdot \boldsymbol{k}=0$. Then $\boldsymbol{k}$ is called the view direction and the line through $\boldsymbol{p}$ in this direction is called the visual ray. Let $\boldsymbol{p} \in M$, then the corresponding point $\boldsymbol{q}$ of the image plane satisfies $\boldsymbol{p}=\boldsymbol{q}+\lambda \boldsymbol{k}$, where $\lambda$ is the signed distance from the image plane to the point $\boldsymbol{p}$. It follows that $\lambda=\boldsymbol{p} \cdot \boldsymbol{k}$, so that we have $\boldsymbol{q}=\boldsymbol{p}-(\boldsymbol{p} \cdot \boldsymbol{k}) \boldsymbol{k}$. The interesting point on $M$ is the point $\boldsymbol{p}$ at where the visual ray is tangent to $M$. These are the points where, viewed in the direction $\boldsymbol{k}$, the surface appears to fold, or to have a boundary or occluding contour. The contour generator $\Gamma$ on $M$ is the set of points of $M$ for which $\boldsymbol{n} \cdot \boldsymbol{k}=0$, where $\boldsymbol{n}$ is the normal vector field of $M$. The corresponding apparent contour $\boldsymbol{\gamma}$ is the set of points $\boldsymbol{q}$ of the image plane forming the projection of $\Gamma$ in the direction $\boldsymbol{k}$ to the image plane. In other word, the contour generator is the singular set of the orthogonal projection restricted on $M$ and the apparent contour is the critical value set. As an application of the standard jet transversality theorem and the theorem of Whitney's plane to plane mapping, we can show that the apparent contour has only 3/2-cusps as generic singularities. Then we have the following proposition.

Proposition 5.10 The apparent contour $\boldsymbol{\gamma}$ is smooth at $\boldsymbol{q}$ except when the view direction $\boldsymbol{k}$ is asymptotic at the corresponding point $\boldsymbol{p}$ of $\Gamma$. The apparent contour has a $3 / 2$-cusp when the line through $\boldsymbol{q}$ in the view direction $\boldsymbol{k}$ is asymptotic and in fact has exactly 3-point contact with $M$ at $\boldsymbol{q}$.

Here the direction of a tangent vector $\boldsymbol{v}$ is asymptotic if it is contained in the kernel direction of the second fundamental form.

We can recognize the cusp point of the apparent contour on the picture of a mountain (cf., Fig. 5.5 : Mt. Moiwa in Sapporo).

Around the smooth point of the apparent contour, we have the following formula for Gauss curvature of Koenderink [43]. 
Theorem 5.11 Assume that the apparent contour is smooth at $\boldsymbol{q}$. Let $\kappa^{q}$ be the curvature of the apparent contour at $\boldsymbol{q}$ and $\kappa^{t}$ is the normal curvature of $M$ at $\boldsymbol{p}$ in the direction of the tangent vector $\boldsymbol{k}$. Then the Gauss curvature $K$ of $M$ at $\boldsymbol{p}$ is given by $K=\kappa^{q} \kappa^{t}$.

This theorem says that if the surface looks convex (respectively, concave) around a point $\boldsymbol{p}$, then the Gauss curvature $K$ at $\boldsymbol{p}$ is positive (respectively, negative). We do not need to touch the surface to discern between positively curved parts and negatively curved parts(cf., Fig. 5.5, Fig. 5.6).

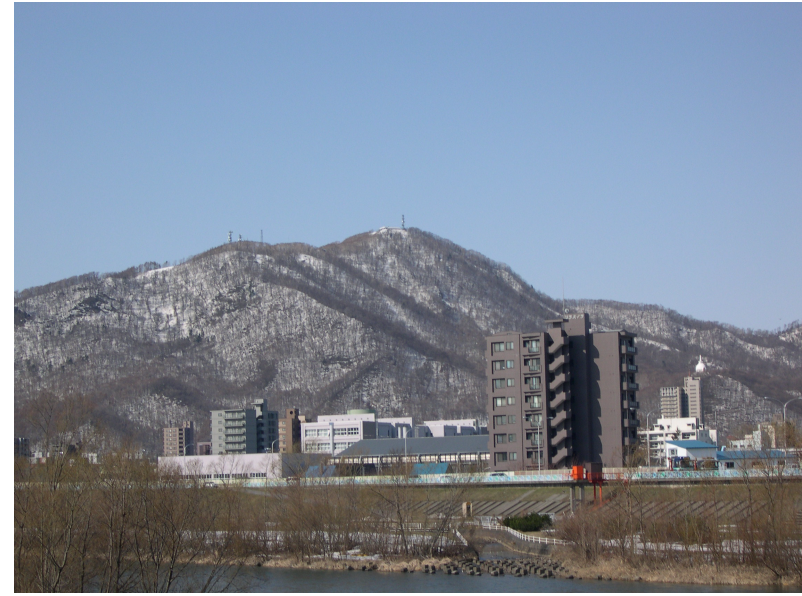

Fig. 5.5

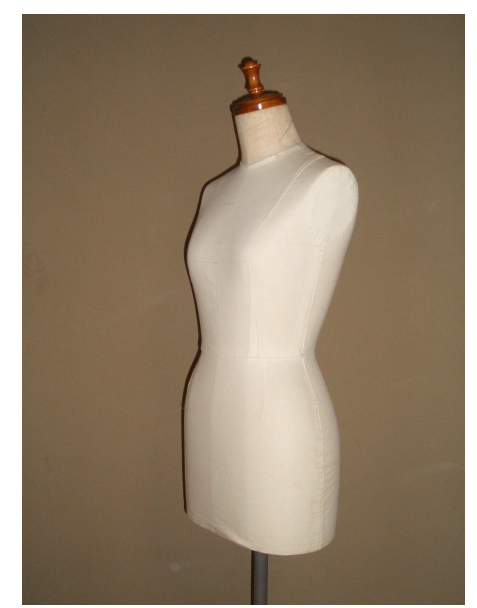

Fig. 5.6

We also have several results on submanifolds of pseudo-spheres in Minkowski space as applications of the theory of singularities[29, 31, 32, 33, 34, 35, 36, 37, 38, 40, 41].

\section{Singularities of solutions for first order partial differ- ential equations}

In this section we consider the following two similar Cauchy problems :

$$
(\mathrm{H}) \quad\left\{\begin{array} { l } 
{ \frac { \partial y } { \partial t } + H ( \frac { \partial y } { \partial x } ) = 0 ( t > 0 ) } \\
{ y ( 0 , x ) = \phi ( x ) , }
\end{array} \quad ( \mathrm { C } ) \quad \left\{\begin{array}{l}
\frac{\partial y}{\partial t}+\frac{\partial f(y)}{\partial x}=0(t>0) \\
y(0, x)=\phi(x),
\end{array}\right.\right.
$$

where $H, f, \phi$ are $C^{\infty}$-functions. The equation $(\mathrm{H})$ is called a Hamilton-Jacobi equation which plays an important role in geometric optics, calculus of variations, optimal control theory and classical mechanics. The equation $(\mathrm{C})$ is called a single conservation law which plays also an important role in gas dynamics and oilreservoir problems. In this article we do not mention how these equations are used in each field. We now solve these Cauchy problems by using the classical method of characteristics. The integration of a first order partial differential equation reduces to the integration of a system of ordinary differential equations, so-called the characteristic equations. We now have a system of ordinary differential equations :

$$
t^{\prime}(\tau)=1, x^{\prime}(\tau)=H^{\prime}(p(\tau)), y^{\prime}(\tau)=-H(p(\tau))+p(\tau) H^{\prime}(p(\tau)), p^{\prime}(\tau)=0, s^{\prime}(\tau)=0 .
$$


This is called the characteristic equation for $(\mathrm{H})$. We consider the initial condition $t(0)=$ $0, x(0)=u, y(0)=\phi(u), p(0)=\phi^{\prime}(u), s(0)=-H(p(0))$ corresponding to the initial curve $\gamma(u)=\Gamma(0, u)$. In this case we have the exact solution

$$
\begin{aligned}
& t(\tau, u)=\tau, x(\tau, u)=u+\tau H^{\prime}\left(\phi^{\prime}(u)\right), y(\tau, u)=\tau\left\{-H\left(\phi^{\prime}(u)\right)+\phi^{\prime}(u) H^{\prime}\left(\phi^{\prime}(u)\right)\right\}+\phi(u), \\
& p(\tau, u)=\phi^{\prime}(u), s(\tau, u)=-H\left(\phi^{\prime}(u)\right) .
\end{aligned}
$$

We call the solution curve of the characteristic equation the characteristics. We now study the properties of the characteristics. We have an embedding $L_{\phi}: \mathbb{R}^{2} \longrightarrow \mathbb{R}^{5}$ given by

$$
L_{\phi}(\tau, u)=(\tau, x(\tau, u), y(\tau, u),-H(p(\tau, u)), p(\tau, u)) .
$$

We also consider the canonical projection $\pi_{2}(t, x, y, s, p)=(t, x)$ and the composition $\Phi(\tau, u)=$ $\pi_{2} \circ L_{\phi}(\tau, u)=(\tau, x(\tau, u))$. Then the Jacobian matrix of $\Phi$ is degenerate at the point $(t, u)$ where $1+\tau H^{\prime \prime}\left(\phi^{\prime}(u)\right) \phi^{\prime \prime}(u)=0$. Assume that $1+\tau H^{\prime \prime}\left(\phi^{\prime}(u)\right) \phi^{\prime \prime}(u) \neq 0$ at a point $\left(\tau_{0}, u_{0}\right)$, then there exists an inverse mapping $\Psi(t, x)$ of $\Phi$ around the point $\left(\tau_{0}, u_{0}+\tau_{0} H^{\prime}\left(\phi^{\prime}\left(u_{0}\right)\right)\right.$ by the inverse mapping theorem. In this case the inverse mapping has the form $\Psi(t, x)=(t, \psi(t, x))$. Moreover we define a function $g$ by $g(t, x)=y(t, \psi(t, x))$. The partial derivatives of $g(t, x)$ can be calculated as $\frac{\partial g}{\partial t}=-H\left(\phi^{\prime}(u)\right), \frac{\partial g}{\partial x}=\phi^{\prime}(u)$, where $(t, x)=\left(\tau, u+\tau H^{\prime}\left(\phi^{\prime}(u)\right)\right)$. It is clear that $g$ is a solution of Hamilton-Jacobi equation $(\mathrm{H})$. For sufficiently short time from the initial time, there always exists the inverse of $\Phi$, so that the smooth (classical) solution of $(\mathrm{H})$ exists.

On the other hand, we also have the characteristic equation for $(\mathrm{C})$. In this case the characteristic equation is the system of equations on 3-dimensional space $\mathbb{R}^{3}$ with the coordinates $(t, x, y)$ given as follows :

$$
t^{\prime}(\tau)=1, x^{\prime}(\tau)=f^{\prime}\left(y(\tau, x(\tau)), y^{\prime}(\tau)=0 .\right.
$$

The corresponding initial condition is $x(0)=u, y(0, x(0))=\phi(u)$. We also have the exact solution as follows :

$$
t(\tau, u)=\tau, x(\tau, u)=u+\tau f^{\prime}(\phi(u)), y(\tau, u)=y(0, x(0))=\phi(u) .
$$

we can also consider an embedding $\mathcal{I}: \mathbb{R}^{2} \rightarrow \mathbb{R}^{3}$ given by

$$
\mathcal{I}(\tau, u)=\left(\tau, u+\tau f^{\prime}(\phi(u)), \phi(u)\right) .
$$

Like as the case for $(\mathrm{H})$, we consider the mapping $\Phi(\tau, u)=\left(\tau, u+\tau f^{\prime}(\phi(u))\right)$. The Jacobian determinant is given by $1+\tau f^{\prime \prime}(\phi(u)) \phi^{\prime}(u)$. Assume that $1+\tau f^{\prime \prime}(\phi(u)) \phi^{\prime}(u) \neq 0$ at a point $\left(\tau_{0}, u_{0}\right)$. By the inverse mapping theorem, there exits the inverse mapping $\Psi(t, x)$ of $\Phi$ around the point $\left(\tau_{0}, u_{0}+\tau_{0} f^{\prime}\left(\phi\left(u_{0}\right)\right)\right.$ with the form $\Psi(t, x)=(t, \psi(t, x))$. We can also calculate the partial derivatives of $\psi$ and show that $g(t, x)=\phi \circ \psi(t, x)$ is a solution of (C). We also have the smooth (classical) solution for sufficiently short time from the initial time. Both of these arguments for $(\mathrm{H})$ and $(\mathrm{C})$ are usually written in the first part of the text book on the theory of partial differential equations[3]. However, there exists a critical time at when the Jacobian determinant vanishes in general. After the critical time, the characteristics on $(t, x)$ plane cross. This means that the solution $y$ is multi-valued. The situation is depicted in Fig. 6.1 and Fig. 6.2. Fig. 6.1 is the picture of the image of $\Pi \circ L_{\phi}$ for $(\mathrm{H})$ with the Hamiltonian function is $H(p)=p^{2}$, where $\Pi(t, x, y, s, p)=(t, x, y)$. Fig. 6.2 is the picture of the image of $\mathcal{I}$ for $(\mathrm{C})$ with $f(y)=y^{2}$. We adopt $\phi(u)=\sin u$ as the initial condition in both cases. 
These are the pictures of graphs of multi-valued solutions solved by the characteristic method. We can observe that the multi-valued solutions appear after some critical times. Moreover, the graph of multi-valued solution for $(\mathrm{C})$ is a smooth surface in $\mathbb{R}^{3}$ and the graph of multi-valued solution for $(\mathrm{H})$ has singularities. Therefore, we can understand the difference of these similar equations by observing the multi-valued solutions for both equations.

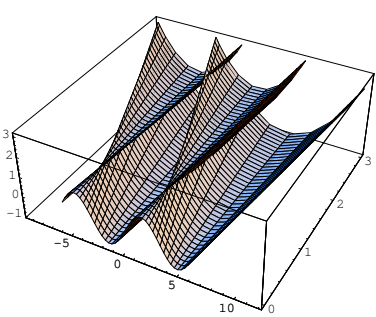

Fig. 6.1

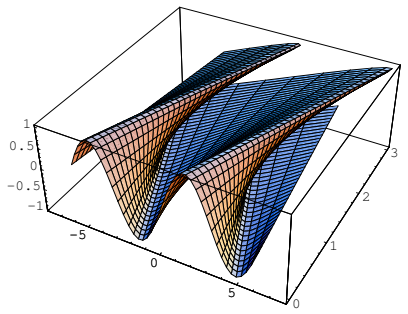

Fig. 6.2

As we mentioned in the above sentences, we have a multi-valued solution if we solve the first order partial differential equation by the characteristic method.

Why these solutions are different?

Since the multi-valued solution for $(\mathrm{C})$ us a smooth embedded surface in $\mathbb{R}^{3}$. The projection of such a surface onto the $(x, t)$-plane $\Phi(\tau, u)$ is the Whitney's plane to plane mapping for generic initial data $\phi(u)$. However, for the multi-valued solution for $(H)$, we have the canonical contact structure $\theta=d y-s d t-p d x$ on $\mathbb{R}^{5}$. The surface $L_{\phi}$ is a smooth embedded surface in $\mathbb{R}^{5}$ with $L_{\phi}^{*} \theta=0$, so that $L_{\phi}$ is a Legendrian submanifold of $\left(\mathbb{R}^{5}, \theta\right)$. In this case the graph of the multi-valued solution $\pi\left(L_{\phi}\right)$ is the wave front of $L_{\phi}$. By Theorem 4.8, generic singularities of the wave fronts in $\mathbb{R}^{3}$ are the cuspidaledge or the swallowtail. We can observe the swallowtails in Fig. 6.1. Therefore the theory of Legendrian singularities is useful for the study of the singularities of multi-valued solutions for first order partial differential equations.

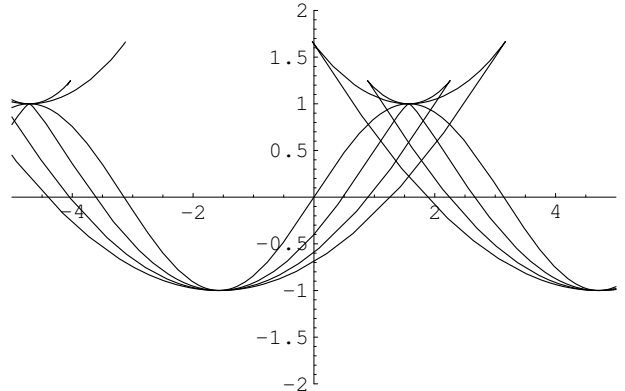

Fig. 6.3

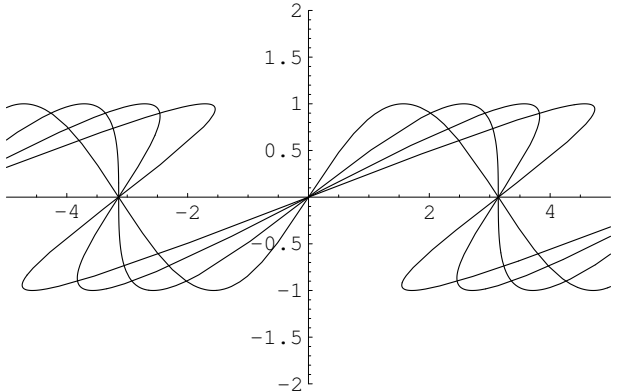

Fig. 6.4

On the other hand, we need single-valued solutions for applications in some area. In this case, we cannot expect the differentiability of the solutions. We call such a solution a weak solution. We call the set of non-smooth points of a weak solution a shock wave of the solution. The notion of viscosity solutions[12] (respectively, entropy solutions[60]) has provided the right weak setting for the study of $(\mathrm{H})$ (respectively, $(\mathrm{C})$ ). Existence and uniqueness of the solution of both solutions hold. Although the existence of both solutions can be proved by the common 
method (i.e., so called the vanishing viscosity method), their features are quite different. It has been known that the viscosity solution for $(\mathrm{H})$ is a piecewise smooth continuous function and the entropy solution for $(\mathrm{C})$ is a piecewise smooth discontinuous function. We can observe their difference on the picture of graphs of multi-valued solutions. Fig. 6.3 and Fig. 6.4 are the pictures of the sections of multi-valued solutions in the plane $(x, t, y)(t=$ constant $)$ after the critical time. We can easily choose a continuous single valued function from Fig. 6.3 (i.e., then minimum branch of the graph of the multi-valued solution). We cannot, however, choose such a function from Fig 6.4.

It is very interesting to study how shock waves appear and propagate. It is obvious that shock waves are deeply related to singularities of multi-valued solutions solved by the characteristic method. The singularity theory of smooth mappings provides the method for studying singularities of multi-valued solutions. In fact, Guckenheimer[16] assumes that the first singular point of the multi-valued solutions for the single conservation law is the Whitney's pleat ( the cusp ), then he describe how shock waves appear for entropy solutions. It is, however, a quite new result that the Whitney's pleat is the first singular point of the multi-valued solution for generic initial data $\phi([20])$. Classifications of generic singularities for multi-valued solutions for general dimensions have been given in the same paper[20]. The history of the study for entropy solutions is much longer than that of viscosity solutions. However, properties of viscosity solutions are well-understood more than those of entropy solutions. One of the reason is that we can easily recognize singularities of the graph of the multi-valued solution for $(\mathrm{H})$.

\section{$7 \quad$ Ruled surfaces}

A surface in Euclidean space is called a ruled surface if it is given by a one-parameter family of lines. Ruled surfaces are classical subjects in differential geometry which have been studied since the 19th century. It has been considered that almost all interesting properties have been already known until the middle of the 20th century. It is, however, paid attention in several areas again [17, 68, 73]. Moreover, the situation is quite different if we consider the case when ruled surfaces have singularities. Generally ruled surfaces have singularities. The first modern study of singularities of ruled surface is given in Cleave's paper on a classification of singularities of developable surfaces of space curves [13] which has appeared incredibly new. After that, there appeared several articles concerning on singularities of developable surfaces in $\mathbb{R}^{3}$ (c.f., $[18,19,22,23,56,71]$ ). On the study of singularities of general ruled surfaces, the authors' paper [24] might be the first result so far as we know.

We now review some basic concepts on classical differential geometry of space curves and ruled surfaces in Euclidean space. For any two vectors $\boldsymbol{x}=\left(x_{1}, x_{2}, x_{3}\right)$ and $\boldsymbol{y}=\left(y_{1}, y_{2}, y_{3}\right)$, we denote $\boldsymbol{x} \cdot \boldsymbol{y}$ as the standard inner product. Let $\boldsymbol{\gamma}: I \longrightarrow \mathbb{R}^{3}$ be a unit speed curve (i.e., $\left\|\boldsymbol{\gamma}^{\prime}(s)\right\|=1$, where $\left.\boldsymbol{\gamma}^{\prime}(s)=d \boldsymbol{\gamma} / d s(s)\right)$. Let us denote $\boldsymbol{t}(s)=\boldsymbol{\gamma}^{\prime}(s)$ and we call $\boldsymbol{t}(s)$ a unit tangent vector of $\boldsymbol{\gamma}$ at $s$. We define the curvature of $\boldsymbol{\gamma}$ by $\kappa(s)=\sqrt{\left\|\boldsymbol{\gamma}^{\prime \prime}(s)\right\|}$. If $\kappa(s) \neq 0$, then the unit principal normal vector $\boldsymbol{n}(s)$ of the curve $\boldsymbol{\gamma}$ at $s$ is given by $\boldsymbol{\gamma}^{\prime \prime}(s)=\kappa(s) \boldsymbol{n}(s)$. The unit vector $\boldsymbol{b}(s)=\boldsymbol{t}(s) \times \boldsymbol{n}(s)$ is called the unit binormal vector of $\boldsymbol{\gamma}$ at $s$. Then we have the Frenet-Serret formulae:

$$
\left\{\begin{array}{l}
\boldsymbol{t}^{\prime}(s)=\kappa(s) \boldsymbol{n}(s) \\
\boldsymbol{n}^{\prime}(s)=-\kappa(s) \boldsymbol{t}(s)+\tau(s) \boldsymbol{b}(s) \\
\boldsymbol{b}^{\prime}(s)=-\tau(s) \boldsymbol{n}(s)
\end{array}\right.
$$

where $\tau(s)=\operatorname{det}\left(\gamma^{\prime}(s), \gamma^{\prime \prime}(s), \gamma^{\prime \prime \prime}(s)\right) /\left\|\gamma^{\prime \prime}(s)\right\|^{2}$ is the torsion of the curve $\gamma$ at $s$. For any unit 
speed curve $\boldsymbol{\gamma}: I \longrightarrow \mathbb{R}^{3}$, we call $\boldsymbol{D}(s)=\tau(s) \boldsymbol{t}(s)+\kappa(s) \boldsymbol{b}(s)$ the Darboux vector field of $\boldsymbol{\gamma}$ (cf., [44], Section 5.2). We define a vector field $\widetilde{\boldsymbol{D}}(s)=(\tau / \kappa)(s) \boldsymbol{t}(s)+\boldsymbol{b}(s)$ along $\boldsymbol{\gamma}$ under the condition that $\kappa(s) \neq 0$ and we call it the modified Darboux vector field of $\boldsymbol{\gamma}$.

On the other hand, a ruled surface in $\mathbb{R}^{3}$ is (locally) the map $F_{(\gamma, \delta)}: I \times \mathbb{R} \rightarrow \mathbb{R}^{3}$ defined by $F_{(\gamma, \delta)}(t, u)=\gamma(t)+u \boldsymbol{\delta}(t)$, where $\boldsymbol{\gamma}: I \rightarrow \mathbb{R}^{3}, \boldsymbol{\delta}: I \rightarrow \mathbb{R}^{3} \backslash\{0\}$ are smooth mappings. We call $\boldsymbol{\gamma}$ the base curve and $\boldsymbol{\delta}$ the director curve. The straight lines $u \mapsto \gamma(t)+u \boldsymbol{\delta}(t)$ are called rulings. We say that a ruled surface $F_{(\gamma, \widetilde{\delta})}$ is a developable surface if the Gaussian curvature $K$ of the regular part of $F_{(\gamma, \widetilde{\delta})}$ is identically zero. The following theorem is the classification theorem of non singular developable surfaces which has been classically known (cf., [77]):

Theorem 7.1 Let $F_{(\gamma, \widetilde{\delta})}$ be a developable surface. Then we always have one of the following situations:

(1) $F_{(\gamma, \widetilde{\delta})}$ is a part of a cylindrical surface (i.e., $\widetilde{\boldsymbol{\delta}}(t)$ is constant).

(2) $F_{(\gamma, \widetilde{\delta})}$ is a part of a conical surface (i.e., $\gamma(t)$ is constant $)$.

(3) $F_{(\gamma, \widetilde{\delta})}$ is a part of a tangent developable ( i.e., $\widetilde{\boldsymbol{\delta}}(t)$ is parallel to the tangent line of $\boldsymbol{\gamma}(t)$ ).

(4) The glue of the above three surfaces.

We have the following examples of developable surfaces:

Example 7.2 (Tangent developables of space curves) Let $\gamma: I \longrightarrow \mathbb{R}^{3}$ be a regular curve (i.e., $\boldsymbol{\gamma}^{\prime}(t) \neq 0$ ). If we choose $\boldsymbol{\delta}(t)=\boldsymbol{\gamma}^{\prime}(t)$, then we call $F_{(\gamma, \delta)}$ the tangent developable of $\gamma$. It has been classically known that the tangent developable of a space curve has the cuspidal edge along the curve $\gamma(t)$ if the torsion $\tau(t) \neq 0$ (cf., Fig. 7.1). It is incredible that the generic classification of the singularities of tangent developables was shown quite recently. Cleave [13] has shown that the tangent developable of a space curve is locally diffeomorphic to $C C R$ (cf., Fig. 7.1) at a point $\gamma\left(t_{0}\right)$ if $\tau\left(t_{0}\right)=0$ and $\tau^{\prime}\left(t_{0}\right) \neq 0$. These conditions are generic for space curves.

Here, $C C R=\left\{\left(x_{1}, x_{2}, x_{3}\right) \mid x_{1}=u^{3}, x_{2}=u v^{3}, x_{3}=v^{2}\right\}$ is the cuspidal crosscap.

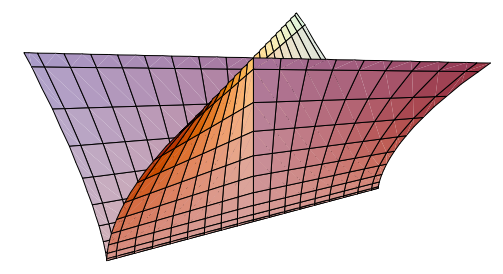

cuspidal crosscap

Fig. 7.1

On the other hand, even if there exists a point $t_{0} \in I$ such that $\gamma^{\prime}\left(t_{0}\right)=0$, we can smoothly extend the tangent vector field along the curve under a certain condition (cf., [19]). Here, we only consider an example given by $\gamma(t)=\left(t^{2}, t^{3}, t^{4}\right)$. In this case $\gamma^{\prime}(t) \neq 0$ except at the origin. The direction of $\boldsymbol{\gamma}^{\prime}(t)$ is equal to the direction of the vector $\boldsymbol{\delta}(t)=\left(2,3 t, 4 t^{2}\right)$ which is 
also smooth at the origin. So the ruled surface $F_{(\gamma, \delta)}$ is called a tangent developable surface of the singular curve $\gamma(t)=\left(t^{2}, t^{3}, t^{4}\right)$. Arnol'd [1] make the observation that this surface has a swallowtail at the origin. It is, however, known that the curve $\gamma(t)=\left(t^{2}, t^{3}, t^{4}\right)$ is deformed into a regular curve under a sufficiently small perturbation. Therefore, the swallowtail is not a generic (stable) singularity of tangent developable surfaces of space curves. For the curve $\gamma(t)=\left(t^{2}, t^{3}, t^{4}\right)$, the tangent developable is given by $F_{(\gamma, \delta)}(t, u)=\left(t^{2}+2 u, t^{3}+3 t u, t^{4}+4 t u\right)$. If we slightly perturb the curve into $\gamma_{\varepsilon}(t)=\left(t^{2}, t^{3}-\varepsilon t, t^{4}\right)$, the corresponding tangent developable is $F_{\left(\gamma_{\varepsilon}, \delta\right)}(t, u)=\left(t^{2}+2 t u, t^{3}-\varepsilon t+u\left(3 t^{2}-\varepsilon\right), t^{4}+4 t^{3} u\right)$, which has a cuspidal crosscap. The situation is depicted in Fig. 7.2. The left picture is $F_{(\gamma, \delta)}(t, u)$ and the right one is $F_{\left(\gamma_{0.5}, \delta\right)}(t, u)$.
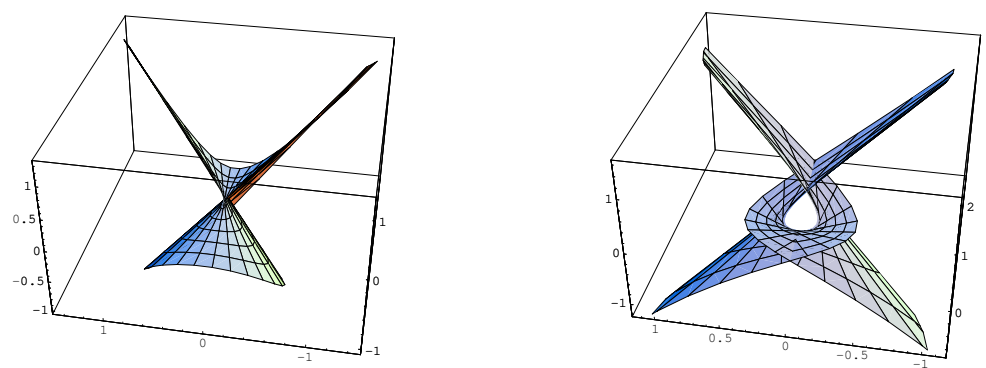

Fig. 7.2

Example 7.3 (Rectifying developables of space curves). We again consider a unit speed regular curve $\gamma: I \longrightarrow \mathbb{R}^{3}$ with non vanishing curvature $\kappa(s)$. There is another important developable surface along $\gamma$ with respect to the Frenet frame. The envelope of the family of rectifying planes along $\boldsymbol{\gamma}$ is called the rectifying developable of the curve $\boldsymbol{\gamma}$. Here, the rectifying plane at $\gamma(s)$ is defined to be the plane generated by the tangent vector $\gamma^{\prime}(s)$ and the binormal vector $\boldsymbol{b}(s)$. In [22] we studied the singularities of rectifying developables of space curves and their geometric meaning. In classical treatises of differential geometry, the Darboux vector of $\boldsymbol{\gamma}$ is defined by $\boldsymbol{D}(s)=\tau(s) \boldsymbol{\gamma}^{\prime}(s)+\kappa(s) \boldsymbol{b}(s)$. However, we define a vector $\tilde{\boldsymbol{D}}(s)=(\tau / \kappa)(s) \boldsymbol{\gamma}^{\prime}(s)+\boldsymbol{b}(s)$ which is called a modified Darboux vector of $\boldsymbol{\gamma}$. We can show that the rectifying developable of a unit speed space curve $\boldsymbol{\gamma}$ is $F_{(\gamma, \tilde{D})}(s, u)=\gamma(s)+u \tilde{\boldsymbol{D}}(s)$, which has already been given in $\S 2$. In [22] we have studied the singularities of the rectifying developable of a space curve $\gamma(s)$ with the condition that $\kappa(s) \neq 0$ and $\tau(s) \neq 0$ for all $s$. It has been shown that the singularities of the rectifying developable of a generic curve with the condition that $\kappa(s) \neq 0$ are the cuspidal edge or the swallowtail.

The swallowtail point of the rectifying developable corresponds to a point $\gamma\left(s_{0}\right)$ where the conditions

$$
\left(\frac{\tau}{\kappa}\right)^{\prime}\left(s_{0}\right) \neq 0,\left(\frac{\tau}{\kappa}\right)^{\prime \prime}\left(s_{0}\right)=0,\left(\frac{\tau}{\kappa}\right)^{(3)}\left(s_{0}\right) \neq 0
$$

are satisfied.

Example 7.4 (Focal developables of space curves).Let $\gamma: I \longrightarrow \mathbb{R}^{3}$ be a regular curve such that the curvature and the torsion of the curve do not vanish at any point. The envelope of the family of normal planes along $\gamma$ is called the focal developable (or the polar developable) 
of the curve $\boldsymbol{\gamma}$. In order to represent the focal developable in our form, we now consider the arclength parameter $s$, so that the tangent vector $\gamma^{\prime}(s)$ is a unit vector. The principal normal of $\boldsymbol{\gamma}$ is $\boldsymbol{n}(s)=\frac{\boldsymbol{\gamma}^{\prime \prime}(s)}{\|\boldsymbol{\gamma}(s)\|}$ and the binormal is $\boldsymbol{b}(s)=\boldsymbol{\gamma}(s) \wedge \boldsymbol{n}(s)$. We denote $\tau(s)$ the torsion of $\gamma(s)$. We now define new curves $\boldsymbol{\sigma}(s)=\gamma(s)+\frac{1}{\kappa(s)} \boldsymbol{n}(s)$ and $\boldsymbol{\delta}(s)=-\frac{\kappa^{\prime}(s)}{\tau(s) \kappa^{2}(s)} \boldsymbol{b}(s)$. Then the focal developable is the surface $F_{(\sigma, \delta)}$ (cf., [44]). The set of singularities is the locus of the centers of osculating spheres. We remember that the osculating sphere of the curve is the sphere which has at least four point contact with the curve. Porteous [65] has shown that the singularities of the focal developable of the generic space curve are the cuspidal edge or the swallowtail. The swallowtail of the focal developable corresponds to the point $\gamma\left(s_{0}\right)$ at where

$$
\frac{\tau(s)}{\kappa(s)}=\left(\frac{\kappa^{\prime}(s)}{\kappa^{2}(s) \tau(s)}\right)^{\prime},\left(\frac{\tau(s)}{\kappa(s)}\right)^{\prime} \neq\left(\frac{\kappa^{\prime}(s)}{\kappa^{2}(s) \tau(s)}\right)^{\prime \prime} .
$$

Under the assumption that $\kappa(s) \neq 0$ and $\tau(s) \neq 0$, the curve $\gamma$ is a spherical curve if and only if $\tau(s) / \kappa(s) \equiv\left(\kappa^{\prime}(s) / \kappa^{2}(s) \tau(s)\right)^{\prime}$. Moreover the swallowtail point of the focal developable corresponds to the point on the curve $\gamma$ at where the curve has exactly five point contact with the osculating sphere.

Example 7.5 (Darboux developables of space curves). We consider the Darboux developable $F_{\left(b, \gamma^{\prime}\right)}(s, u)=\boldsymbol{b}(s)+u \boldsymbol{\gamma}^{\prime}(s)$ of $\boldsymbol{\gamma}$ with $\kappa(s) \neq 0$. A point $\left(s_{0}, u_{0}\right)$ is a singular point of the Darboux developable of $\gamma$ if and only if $u_{0}=(\tau / \kappa)\left(s_{0}\right)$. In [22], it has been shown that the Darboux developable of a generic space curve is locally diffeomorphic to the cuspidal edge or the swallowtail around a singular point under the condition that $\tau(s) \neq 0$. The swallowtail point of the Darboux developable corresponds to a point $\gamma\left(s_{0}\right)$ where the conditions

$$
\left(\frac{\tau}{\kappa}\right)^{\prime}\left(s_{0}\right)=0,\left(\frac{\tau}{\kappa}\right)^{\prime \prime}\left(s_{0}\right) \neq 0
$$

are satisfied.

On the other hand, a curve $\gamma(s)$ with constant $(\tau / \kappa)(s)$ is a cylindrical helix. So the singularities of Darboux developables describe how the shape of $\gamma(s)$ is similar to cylindrical helices. In fact, Fig. 7.3 is the picture of the Darboux developable of $\gamma(t)=\left(t, t^{2}, t^{4}\right)$. We can observe the cuspidal crosscap.

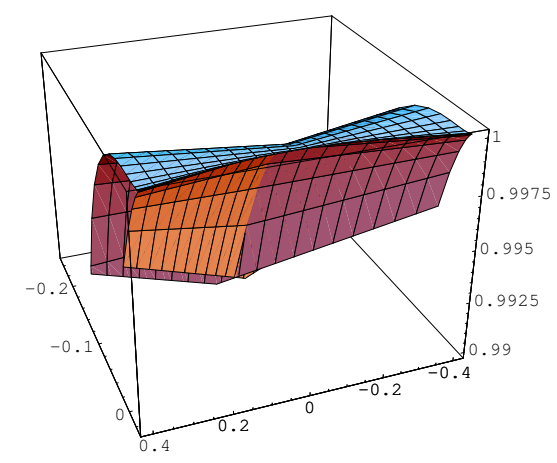

Fig. 7.3. 
It is known that the Darboux developable is locally diffeomorphic to $C C R$ at the point $s$ where $\tau(s)=0$ for generic $\boldsymbol{\gamma}$.

We now give examples of ruled surfaces which are not developable surfaces. Let $\gamma(s)$ be a unit speed space curve with $\kappa(s) \neq 0$.

Example 7.6 (The principal normal surface of a space curve) A ruled surface

$$
F_{(\gamma, n)}(s, u)=\gamma(s)+u \boldsymbol{n}(s)
$$

is called the principal normal surface of $\boldsymbol{\gamma}$. By the Frenet-Serret formulae, we can show that

$$
\boldsymbol{\gamma}^{\prime}(s) \times \boldsymbol{n}(s)+u \boldsymbol{n}^{\prime}(s) \times \boldsymbol{n}(s)=(1-u \kappa(s)) \boldsymbol{b}(s)-\tau(s) u \boldsymbol{t}(s) .
$$

Therefore $\left(s_{0}, u_{0}\right)$ is a singular point of $F_{(\gamma, n)}$ if and only if $\tau\left(s_{0}\right)=0$ and $u_{0}=\frac{1}{\kappa\left(s_{0}\right)}$.

Consider the space curve defined by $\gamma(t)=\left(t, t^{2}, t^{4}\right)$. In this case, we can calculate that the principal normal direction is given by

$$
\widetilde{\boldsymbol{n}}(t)=\left(-24 t^{5}-2,1-32 t^{6}, 6 t^{2}+16 t^{4}\right) .
$$

Therefore, the principal normal surface is given by

$$
F(t, u)=\left(t-u\left(24 t^{5}+2\right), t^{2}+u\left(1-32 t^{6}\right), t^{4}+u\left(6 t^{2}+16 t^{4}\right)\right) .
$$

We can draw the picture of the surface by using the graphical tool of Mathematica in Fig. 7.4. The singular point looks like a cross cap.
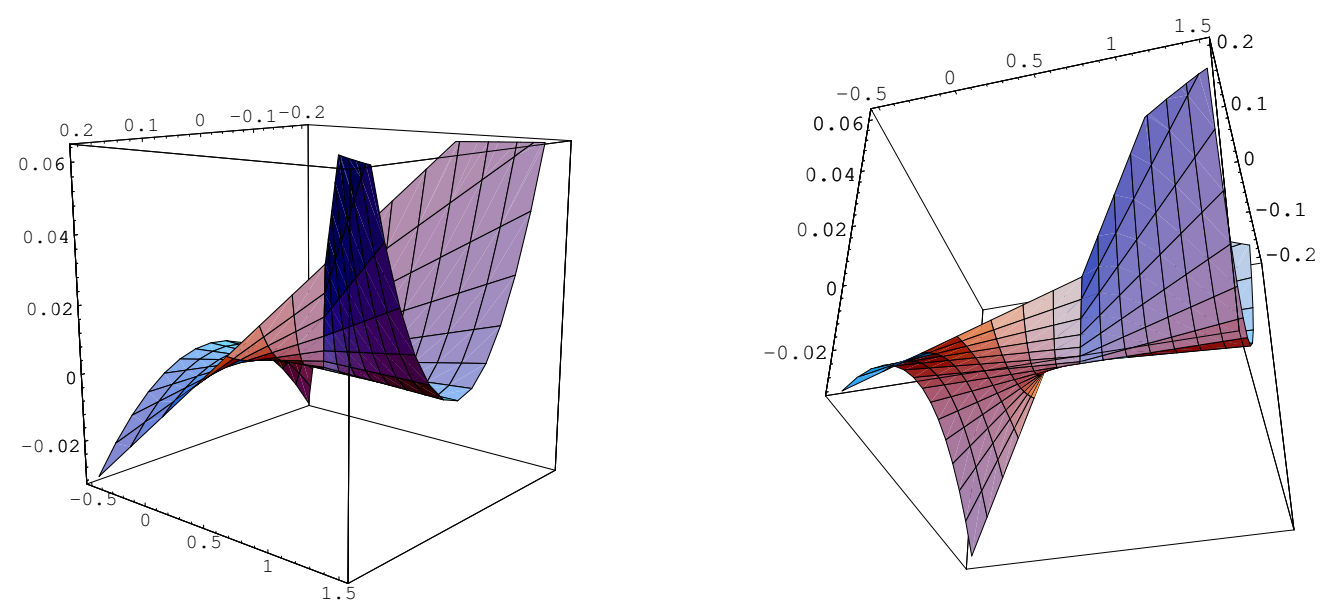

Fig. 7.4

For principal normal surfaces, we have the following classification theorem. 
Theorem 7.7 For a unit speed curve $\boldsymbol{\gamma}: I \longrightarrow \mathbb{R}^{3}$ with $\kappa(s) \neq 0$, the principal normal surface $F_{(\gamma, n)}(s, u)$ of $\gamma$ is a cross cap at $\left(s_{0}, u_{0}\right)$ if and only if

$$
u_{0}=\frac{1}{\kappa\left(s_{0}\right)}, \tau\left(s_{0}\right)=0 \text { and } \tau^{\prime}\left(s_{0}\right) \neq 0 .
$$

We now consider singularities of developable surfaces. We have the following simple lemma.

Lemma 7.8 Let $F_{(\gamma, \delta)}$ be a noncylindrical ruled surface (i.e., $\boldsymbol{\delta}(t) \times \boldsymbol{\delta}^{\prime}(t) \neq 0$ ). Then $F_{(\gamma, \delta)}$ is a developable surface if and only if there exist smooth functions $\mu, \lambda: I \longrightarrow \mathbb{R}$ such that $\gamma^{\prime}(t)=\mu(t) \boldsymbol{\delta}(t)+\lambda(t) \boldsymbol{\delta}^{\prime}(t)$.

By the lemma, we can control noncylindrical developable surfaces by using $\mu(t), \lambda(t)$ and $\boldsymbol{\delta}(t)$. We define the space of noncylindrical developable surfaces as follows:

$$
\operatorname{Dev}\left(I, \mathbb{R}^{3}\right)=\left\{(\mu, \lambda, \boldsymbol{\delta}) \mid(\mu, \lambda, \boldsymbol{\delta}): I \longrightarrow \mathbb{R}^{2} \times\left(\mathbb{R}^{3} \backslash\{0\}\right) C^{\infty} \text { map with } \boldsymbol{\delta}(t) \wedge \boldsymbol{\delta}^{\prime}(t) \neq 0\right\}
$$

with Whitney $C^{\infty}$ topology. As an application of the classification of singularities of the tangent developable of a (not necessary regular) space curve (cf., $[13,18,19,56,71]$ ), we can state a classification theorem of singularities of generic developable surfaces.

Theorem 7.9 Let $F_{(\gamma, \delta)}: I \times J \longrightarrow \mathbb{R}^{3}$ be a noncylindrical developable surface. We fix smooth functions $\mu, \lambda: I \longrightarrow \mathbb{R}$ with $\gamma^{\prime}(t)=\mu(t) \boldsymbol{\delta}(t)+\lambda(t) \boldsymbol{\delta}^{\prime}(t)$. Let $\left(t_{0}, u_{0}\right) \in I \times J$ be a singular point of $F_{(\gamma, \delta)}$ and put $x_{0}=\gamma\left(t_{0}\right)+u_{0} \boldsymbol{\delta}\left(t_{0}\right)=F_{(\gamma, \delta)}\left(t_{0}, u_{0}\right)$.

(1) Suppose that $\operatorname{det}\left(\boldsymbol{\delta}\left(t_{0}\right) \boldsymbol{\delta}^{\prime}\left(t_{0}\right) \boldsymbol{\delta}^{\prime \prime}\left(t_{0}\right)\right) \neq 0$. Then

(a) The germ of $F_{(\gamma, \delta)}(I \times J)$ at $x_{0}$ is locally diffeomorphic to $C \times \mathbb{R}$ if $u_{0}=\lambda\left(t_{0}\right)$ and $\mu\left(t_{0}\right) \neq \lambda^{\prime}\left(t_{0}\right)$.

(b) The germ of $F_{(\gamma, \delta)}(I \times J)$ at $x_{0}$ is locally diffeomorphic to $S W$ if $u_{0}=\lambda\left(t_{0}\right), \mu\left(t_{0}\right)=\lambda^{\prime}\left(t_{0}\right)$ and $\mu^{\prime}\left(t_{0}\right) \neq \lambda^{\prime \prime}\left(t_{0}\right)$.

(2) Suppose that $\operatorname{det}\left(\boldsymbol{\delta}\left(t_{0}\right) \boldsymbol{\delta}^{\prime}\left(t_{0}\right) \boldsymbol{\delta}^{\prime \prime}\left(t_{0}\right)\right)=0$. Then The germ of $F_{(\gamma, \delta)}(I \times J)$ at $x_{0}$ is locally diffeomorphic to $C C R$ if $u_{0}=\lambda\left(t_{0}\right), \mu\left(t_{0}\right) \neq \lambda^{\prime}\left(t_{0}\right)$ and $\operatorname{det}\left(\boldsymbol{\delta}\left(t_{0}\right) \boldsymbol{\delta}^{\prime}\left(t_{0}\right) \boldsymbol{\delta}^{(3)}\left(t_{0}\right)\right) \neq 0$.

On the other hand, we now review recent results on singularities of general ruled surfaces (cf., [24]). The Gaussian curvature of the regular part of a ruled surface is generally non-positive. So the developable surface is a member of the special class of ruled surfaces. Therefore we have the following natural question:

Question. How are singularities of developable surfaces different from those of "general" ruled surfaces?

We give a classification of singularities of general ruled surface. Let $C^{\infty}\left(I, \mathbb{R}^{3} \times S^{2}\right)$ be the space of smooth mappings $(\gamma, \delta): I \longrightarrow \mathbb{R}^{3} \times S^{2}$ equipped with Whitney $C^{\infty}$-topology, where $I$ is an open interval. In [24] we have shown the following theorem which gives a "generic" answer to the above question.

Theorem 7.10 There exists an open dense subset $\mathcal{O} \subset C^{\infty}\left(I, \mathbb{R}^{3} \times S^{2}\right)$ such that the germ of the ruled surface $F_{(\gamma, \delta)}$ at any point $\left(t_{0}, u_{0}\right)$ is an immersion germ or $\mathcal{A}$-equivalent to the cross cap for any $(\gamma, \delta) \in \mathcal{O}$. 
It is well known that any singular point for generic smooth mappings from a surface to $\mathbb{R}^{3}$ is the cross cap (cf., $[1,5,13,19]$ ). The set of ruled surfaces is a very small subset in the space of all $C^{\infty}$-mappings. The above theorem, however, asserts that the generic singularities of ruled surfaces are the same as those of $C^{\infty}$-mappings.

We can summarize the results of the above theorem as the following relations :

$\{$ Singularities of generic developable surfaces $\} \neq\{$ Singularities of generic ruled surfaces $\}$,

$\{$ Singularities of generic ruled surfaces $\}=\left\{\right.$ Singularities of generic $C^{\infty}$-mappings $\}$.

We remark that the cross cap is realized as a singularity of a ruled surface as follows: Consider curves $\gamma(t)=\left(t^{2}, 0,0\right)$ and $\delta(t)=\left(0, \frac{1}{\sqrt{1+t^{2}}}, \frac{t}{\sqrt{1+t^{2}}}\right)$, then $F_{(\gamma, \delta)}(t, u)$ is the cross cap (cf., Fig. 4) which corresponds to the normal form.

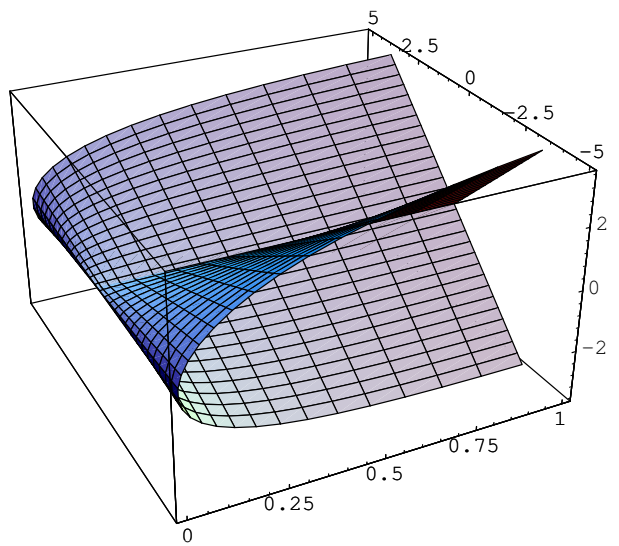

Fig. 7.5

One of the examples of ruled surfaces with cross caps is the Plücker conoid which is given by $\gamma(\theta)=(0,0,2 \cos \theta \sin \theta)$ and $\delta(\theta)=(\cos \theta, \sin \theta, 0)(0 \leq \theta \leq 2 \pi)$ (cf., Fig. 7.6).

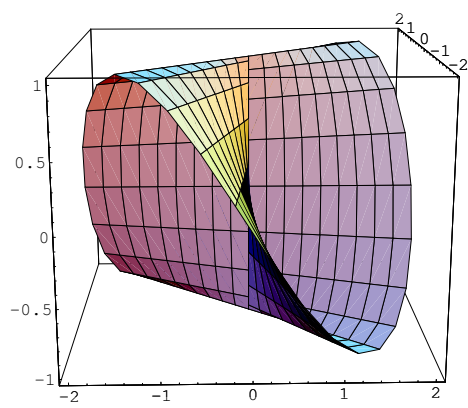

Fig. 7.6 


\section{Gravitational lensings}

Recently there appeared several articles considering gravitation lensing systems as applications of the theory of singularities for smooth mappings. The gravitational lensing is the deflection of light from a distant source (e.g., quasar) by an intervening matter distribution (e.g., a galaxy or a cluster of galaxies). The first gravitational lensed quasar was detected only in 1979. By now gravitational lensing is quite an active field in astrophysics [63, 72].

On the other hand, singularity theory of Lagrangian varieties [2, 79] is the best natural setting for discussing optical systems. In fact, A.O. Petters and his collaborators [61, 63] pointed out that a single gravitation lensing can be described in the framework of symplectic geometry. Especially the caustics in a single gravitation lensing system coincide with caustics in the theory of Lagrangian singularities. Moreover, they also investigate multiplane gravitation lensing as an application of singularity theory [46, 63]. In another paper [47] they pointed out that new, unexpected from the standard singularity theory point of view, caustics might appear for multiplane gravitational lensing. In [30] the symplectic framework for multiplane gravitational lensing based on the notion of symplectic relation was given.

In the first place, we give a quick review of the basic concepts from the theory of gravitational lensing discussed already in [47, 46, 61, 62, 63, 72].

(1) Single lensing (cf., $[61,63,72]$ ) Consider the typical single lens plane gravitational lensing as follows:

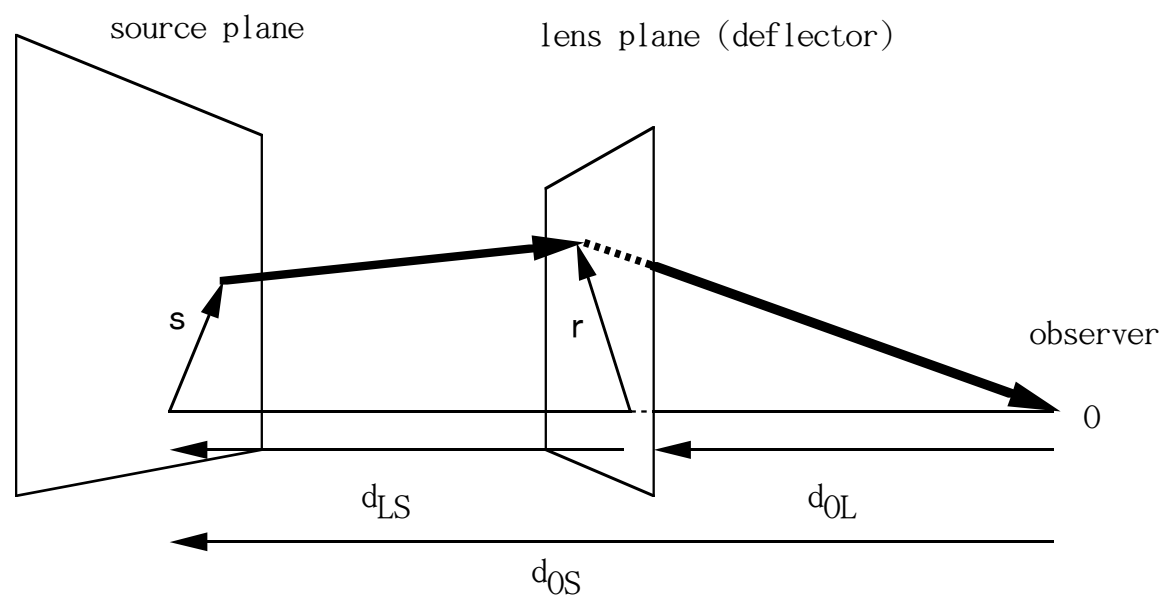

Fig. 8.1

We assume that the deflector is thin and apply the small angle approximation (cf.,[61]). The extra time with respect to the unperturbed ray is giving the time-delay map; $T_{\boldsymbol{s}}: \mathbb{R}^{2} \supset U \longrightarrow \mathbb{R}$ defined by

$$
T_{\boldsymbol{s}}(\boldsymbol{r})=\left(1+z_{L}\right)\left[\frac{d_{O L} d_{O S}}{2 d_{L S}}\left\|\frac{\boldsymbol{s}}{d_{O S}}-\frac{\boldsymbol{r}}{d_{O L}}\right\|^{2}-\Psi(\boldsymbol{r})\right] .
$$

Here, $z_{L}$ is the red shift of the lens plane, $d_{O L}, d_{O S} . d_{L S}$ are angular diameter distances, $\boldsymbol{r}$ is the position on the lens plane where the ray hits, $s$ is the position of the source, and $\Psi(\boldsymbol{r})$ is the two-dimensional potential of the deflector on the lens plane. The deflector potentials $\Psi$ occurring in the time-delay map are given by

$$
\Psi(\boldsymbol{r})=4 \int_{\mathbb{R}^{n}} \sigma\left(\boldsymbol{r}^{\prime}\right) \ln \left\|\frac{\boldsymbol{r}^{\prime}-\boldsymbol{r}}{d_{O L}}\right\| .
$$


They are solutions of two-dimensional Poisson equation $\Delta \Psi(\boldsymbol{r})=8 \pi \sigma(\boldsymbol{r})$, where $\sigma(\boldsymbol{r})$ is the surface mass density. By suitable coordinate transformations, we can express the time-delay map in the convenient form:

$$
T \boldsymbol{y}(\boldsymbol{x})=\Theta_{L}\left[\frac{\|\boldsymbol{x}-\boldsymbol{y}\|^{2}}{2}-\psi(\boldsymbol{x})\right] \quad\left(\boldsymbol{x}, \boldsymbol{y} \in \mathbb{R}^{2}\right)
$$

Here $\boldsymbol{y}$ corresponds to the point on the source $\boldsymbol{s}$-plane and $\boldsymbol{x}$ corresponds to the point on the lens plane $\boldsymbol{r}$.

Fermat's principle yields the critical points of the time delay map $T \boldsymbol{y}(\boldsymbol{x})$ with respect to variations in $\boldsymbol{x}$ determining those rays that are real light rays (cf., [61]). For this reason, a critical point of $T \boldsymbol{y}(\boldsymbol{x})$ relative to $\boldsymbol{x}$ is called an image of the point source at $\boldsymbol{y}$. The magnification of an image $\boldsymbol{x}$ of a source at $\boldsymbol{y}$ is defined by

$$
A \boldsymbol{y}(\boldsymbol{x})=\frac{1}{\left\|\operatorname{det} T_{\boldsymbol{x} \boldsymbol{x}}(\boldsymbol{x} ; \boldsymbol{y})\right\|},
$$

where $T(\boldsymbol{x} ; \boldsymbol{y})=T \boldsymbol{y}(\boldsymbol{x})$ and $T \boldsymbol{x} \boldsymbol{x}(\boldsymbol{x} ; \boldsymbol{y})$ is the Hessian matrix with respect to $\boldsymbol{x}$. A caustic point in gravitational lensing is a position $\boldsymbol{y} \in \mathbb{R}^{2}$ for which a source at $\boldsymbol{y}$ will have at least one image of infinite magnification. In other words, caustics are source positions $\boldsymbol{y} \in \mathbb{R}^{2}$ for which the time-delay map $T \boldsymbol{y}(\boldsymbol{x})$ has at least one degenerate critical point (i.e., $\left.\operatorname{det} T_{\boldsymbol{x}} \boldsymbol{x}(\boldsymbol{x} ; \boldsymbol{y})=0\right)$. So, we may consider that the time-delay map is the generating family of a certain Lagrangian submanifold in $T^{*} \mathbb{R}^{2}$ (cf., $\S 3$ ).

(2) Multiplane gravitational lensing (cf., $[47,62,63,72])$ Although we can consider a general $k$-planes gravitational lensing, we now only consider the case when $k=2$ (i.e, a double plane gravitational lensing) for convenience.

The typical double lens plane gravitational lensing situation is given as follows (Fig. 8.2):

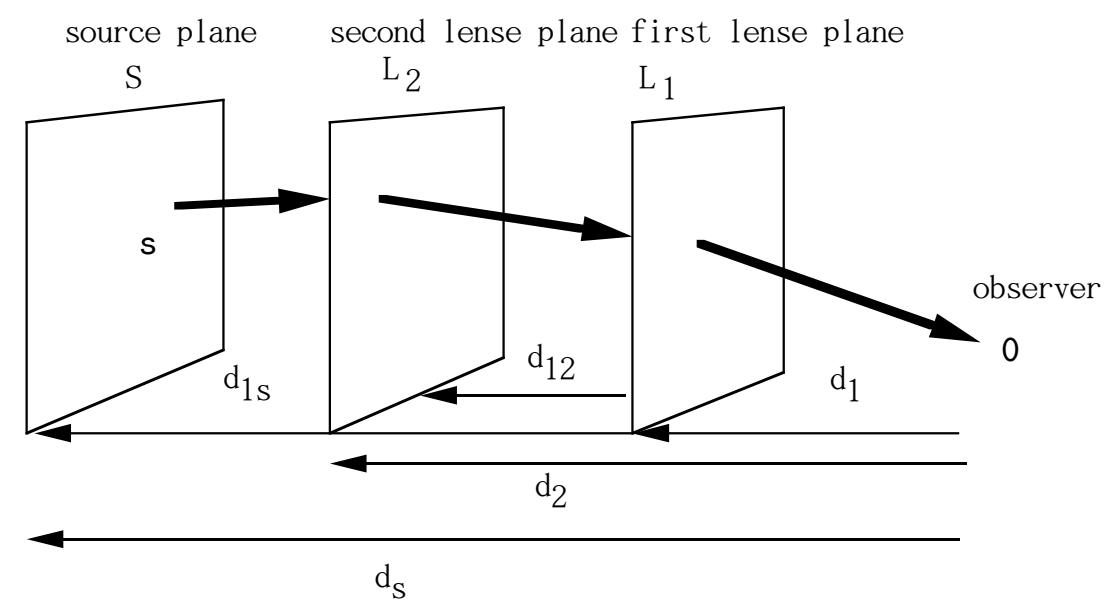

Fig. 8.2

There are two lens planes with "thin" deflectors in each plane. The deflectors are assumed to be independent, that is, the lens planes are sufficiently spaced so that they do not interact. Furthermore, the small angle approximation is assumed. We also parameterize all rays originating from the point source at $\boldsymbol{s}$, deflected by two gravitational lens, using the 4-dimensional vectors $\left(\boldsymbol{r}_{1}, \boldsymbol{r}_{2}\right)$. Relative to these approximations the extra time $T_{\boldsymbol{s}}$ to reach the indicated 
observer from $\boldsymbol{s}$ is given by the time-delay map. It is the function $T_{\boldsymbol{s}}: U_{1} \times U_{2} \subset \mathbb{R}^{4} \rightarrow \mathbb{R}$ with each domain $U_{i} \subset \mathbb{R}^{2}$ being an open subset, defined by

$$
T_{\boldsymbol{s}}\left(\boldsymbol{r}_{1}, \boldsymbol{r}_{2}\right)=\sum_{i=1}^{2}\left(1+z_{i}\right)\left[\frac{d_{i} d_{i+1}}{2 d_{i, i+1}}\left\|\frac{\boldsymbol{r}_{i+1}}{d_{i+1}}-\frac{\boldsymbol{r}_{i}}{d_{i}}\right\|^{2}-\Psi_{i}\left(\boldsymbol{r}_{i}\right)\right]
$$

Here, $z_{i}$ is the redshift of the $i$ the lens plane, $d_{i j}$ is the angular diameter distance separating the $i$ the and $j$ the lens planes, $d_{i}$ is the angular diameter distance from the observer to the $i t h$ lens plane with $d_{k+1} \equiv d_{S}$ the distance to the source plane, $\boldsymbol{r}_{i}$ is the position on the $i$ the plane where the ray hits, $\boldsymbol{r}_{k+1} \equiv \boldsymbol{s}$, and $\Psi_{i}\left(\boldsymbol{r}_{i}\right)$ is the two-dimensional potential of the deflector on the $i$ the lens plane.

By suitable coordinate transformations, the double plane time-delay map can be expressed conveniently as follows:

$T \boldsymbol{y}\left(\boldsymbol{x}_{1}, \boldsymbol{x}_{2}\right)=\Theta_{1}\left[\frac{\left\|\boldsymbol{x}_{2}-\boldsymbol{x}_{1}\right\|^{2}}{2}-\beta_{1,2} \psi_{1}\left(\boldsymbol{x}_{1}\right)\right]+\Theta_{2}\left[\frac{\left\|\boldsymbol{x}_{2}-\boldsymbol{y}\right\|^{2}}{2}-\beta_{2,3} \psi_{2}\left(\boldsymbol{x}_{2}\right)\right],\left(\boldsymbol{x}_{1}, \boldsymbol{x}_{2}, \boldsymbol{y} \in \mathbb{R}^{2}\right)$.

In [62], Fermat's principle has been adapted exactly in the same way as it was used already for the single lens plane case, so that the image of a gravitational lensed point like light source at position $\boldsymbol{y}$ are identified to the critical points of $T \boldsymbol{y}$ e.g., the set of images is given as follows:

$$
\left\{\left(\boldsymbol{x}_{1}, \boldsymbol{x}_{2}\right) \mid \operatorname{grad}_{\boldsymbol{x}_{i}} T \boldsymbol{y}\left(\boldsymbol{x}_{1}, \boldsymbol{x}_{2}\right)=0, i=1,2\right\} .
$$

If we adapt this principle simply, we cannot distinguish the effect of individual lens planes. In fact, in [62] two lens planes are treated like a single lens altogether. It is, however, pointed out in [47] that double-folds or handkerchiefs might appear as the generic caustics for double plane lensings. These singularities do not appear as generic caustics under the above construction.

In [61] Petters pointed out that single gravitational lensing can be described in the framework of symplectic geometry (i.e, Lagrangian singularity theory).

Now let us recall the time-delay map $T \boldsymbol{y}(\boldsymbol{x})$. If we consider the family of functions $F$ : $\mathbb{R}^{2} \times \mathbb{R}^{2} \rightarrow \mathbb{R}$ given by

$$
F\left(\ell_{1}, \ell_{2}, q_{1}, q_{2}\right)=\Theta\left[\frac{\left\|\left(\ell_{1}, \ell_{2}\right)-\left(q_{1}, q_{2}\right)\right\|^{2}}{2}-\psi\left(\ell_{1}, \ell_{2}\right)\right]=T_{q}(\ell)
$$

we can easily verify that $F$ is a Morse family, so $\Phi_{F}: \Sigma(F) \rightarrow T^{*} \mathbb{R}^{2}$ is a Lagrangian immersion. Here, we have

$$
\Sigma(F)=\left\{(\boldsymbol{x}, \boldsymbol{y}) \mid \operatorname{grad}_{\boldsymbol{x}} T=0\right\},
$$

so that the Lagrangian immersion is corresponding to those rays that are actual light rays. The set of critical values of the Lagrangian map $\pi \circ \Phi_{F}$ is the caustic.

On the other hand, we present another symplectic framework for single gravitation lensing, which is essentially the same as the above framework. Our framework will be, however, much useful when we try to generalize this framework to the case of multiple planes gravitational lensing (cf., §4).

We consider the product symplectic space

$$
\mathcal{M}_{(x, y)}=\left(T^{*} M_{x} \times T^{*} M_{y}, \omega_{M_{y}} \ominus \omega_{M_{x}}\right)
$$


where $\omega_{M_{y}}$ and $\omega_{M_{x}}$ are the corresponding canonical symplectic forms, and $\Omega_{(x, y)}=\omega_{M_{y}} \ominus \omega_{M_{x}}=$ $\pi_{M_{y}}^{*} \omega_{M_{y}}-\pi_{M_{x}}^{*} \omega_{M_{x}}$, where $\pi_{M_{x}}, \pi_{M_{y}}$ are the canonical projections of the product $T^{*} M_{x} \times T^{*} M_{y}$. The corresponding phase spaces $\left(T^{*} M_{x}, \omega_{M_{x}}\right)$ and $\left(T^{*} M_{y}, \omega_{M_{y}}\right)$ are called the observer space and the source space respectively. In our 2-dimensional case $M_{x}=\mathbb{R}^{2}$ and $M_{y}=\mathbb{R}^{2}$. The concrete realized single lensing system is represented (following [61]) by the Lagrangian submanifold

$$
L_{\psi}=\left\{\left(\left(\boldsymbol{x}, \operatorname{grad}_{\boldsymbol{x}} T\right),\left(\boldsymbol{y}, \operatorname{grad}_{\boldsymbol{y}} T\right)\right) \mid(\boldsymbol{x}, \boldsymbol{y}) \in M_{x} \times M_{y}\right\} .
$$

This means that the generating function of $L_{\psi}$ is the time-delay map

$$
T(\boldsymbol{x} ; \boldsymbol{y})=\Theta_{L}\left[\frac{\|\boldsymbol{x}-\boldsymbol{y}\|^{2}}{2}-\psi(\boldsymbol{x})\right] \quad\left(\boldsymbol{x}, \boldsymbol{y} \in \mathbb{R}^{2}\right) .
$$

By the previous arguments, light rays are given by $\left\{(\boldsymbol{x}, \boldsymbol{y}) \mid \operatorname{grad}_{\boldsymbol{x}} T=0\right\}$ and the set of point sources for light rays is the Lagrangian submanifold

$$
L_{S}=\left\{\left(\boldsymbol{y}, \operatorname{grad}_{\boldsymbol{y}} T\right) \in T^{*} M_{y} \mid(\boldsymbol{x}, \boldsymbol{y}) \in M_{x} \times M_{y}, \operatorname{grad}_{\boldsymbol{x}} T=0\right\}
$$

of $T^{*} M_{y}$. Then we have

$$
\left.\pi_{M_{y}}\left(L_{\psi} \cap\left(\left(M_{x} \times\{0\}\right) \times T^{*} M_{y}\right)\right)\right)=L_{S} .
$$

Let us recall the basic notions of the theory of symplectic relations ([42]). Let $X_{1}, X_{2}$ be smooth manifolds with the same dimension. We consider the product symplectic manifold

$$
\left(T^{*} X_{1} \times T^{*} X_{2}, \omega_{X_{2}} \ominus \omega_{X_{1}}\right),
$$

where $\omega_{X_{2}} \ominus \omega_{X_{1}}=\pi_{2}^{*} \omega_{X_{2}}-\pi_{1}^{*} \omega_{X_{1}}$. We define a symplectic relation from $T^{*} X_{1}$ to $T^{*} X_{2}$ as a Lagrangian submanifold $R$ of $\left(T^{*} X_{1} \times T^{*} X_{2}, \omega_{X_{2}} \ominus \omega_{X_{1}}\right)$. If the restriction of the projection

$$
\pi_{X_{1}} \times \pi_{X_{2}}: T^{*} X_{1} \times T^{*} X_{2} \longrightarrow X_{1} \times X_{2}
$$

to $R$ is always non-singular, we call $R$ the elementary symplectic relation. Let $R$ be a symplectic relation in $\left(T^{*} X_{1} \times T^{*} X_{2}, \omega_{X_{2}} \ominus \omega_{X_{1}}\right)$ and $S$ be a subset of $T^{*} X_{1}$, then the symplectic image of $S$ by $R$ is defined as

$$
R(S)=\left\{p_{2} \in T^{*} X_{2}: \exists_{p_{1} \in S}\left(p_{1}, p_{2}\right) \in R\right\}
$$

If $S$ is Lagrangian submanifold in $\left(T^{*} X_{1}, \omega_{X_{1}}\right)$, then $R(S)$ is a Lagrangian subset in $\left(T^{*} X_{2}, \omega_{X_{2}}\right)$.

Since both of $S$ and $R$ are Lagrangian submanifolds, we have their generating families at least locally. We only consider the local situation here, so that we assume that $X_{1}=X_{2}=$ $\mathbb{R}^{n}$. Let $F_{1}:\left(\mathbb{R}^{k_{1}} \times X_{1}, 0\right) \longrightarrow \mathbb{R}$ be a generating family of a Lagrangian submanifold germ $S \subset T^{*} X_{1}$ and $F_{2}:\left(\mathbb{R}^{k_{2}} \times\left(X_{1} \times X_{2}\right), 0\right) \longrightarrow \mathbb{R}$ be a generating family of a symplectic relation $R \subset T^{*} X_{1} \times T^{*} X_{2}$. Then we have a function germ

$$
F:\left(\left(\mathbb{R}^{k_{1}} \times X_{1} \times \mathbb{R}^{k_{2}}\right) \times X_{2}, 0\right) \longrightarrow \mathbb{R}
$$

defined by

$$
F\left(\left(\ell_{1}, q_{1}, \ell_{2}\right), q_{2}\right)=F_{1}\left(\ell_{1}, q_{1}\right)+F_{2}\left(\ell_{2}, q_{1}, q_{2}\right)
$$

Hence we have the following lemma: 
Lemma 8.1 If $F$ is a Morse family, then $F$ is a generating family of the Lagrangian submanifold $R(S) \subset T^{*} X_{2}$.

In the case of single gravitational lensing, if $S_{0}$ denotes the observer Lagrangian submanifold of system of gravitational rays then the source Lagrangian submanifold of rays is an image

$$
L_{\psi}\left(S_{0}\right) \subset T^{*} M_{y} .
$$

In the standard setting (cf. [61] and the previous arguments ) $S_{0}$ is the zero section of the cotangent bundle $T^{*} M_{x}$. Therefore we have

$$
L_{\psi}\left(S_{0}\right)=\left\{\left(\boldsymbol{y}, \operatorname{grad}_{\boldsymbol{y}} T\right) \mid \operatorname{grad}_{\boldsymbol{x}} T=0\right\},
$$

so that the generating family for $L_{\psi}\left(S_{0}\right)$ is given by

$$
F\left(\ell_{1}, \ell_{2}, q_{1}, q_{2}\right)=\Theta\left[\frac{\left\|\left(\ell_{1}, \ell_{2}\right)-\left(q_{1}, q_{2}\right)\right\|^{2}}{2}-\psi\left(\ell_{1}, \ell_{2}\right)\right],
$$

which is the same generating family as that of the source Lagrangian submanifold in the previous framework in [61]. We call the pair $(S, R)$ a (single) lensing system if $S$ is a Lagrangian submanifold of $T^{*} X_{1}$ and $R$ is a symplectic relation from $T^{*} X_{1}$ to $T^{*} X_{2}$. If the projection $\left.\pi_{1}\right|_{R}: R \longrightarrow T^{*} X_{1}$ is non-singular, $R$ is the graph of a symplectomorphism $H: T^{*} M_{x} \longrightarrow$ $T^{*} M_{y}$. In this case we say that $(S, R)$ is regular. Moreover, if $S$ is the zero section of $T^{*} X_{1}$, we call $(S, R)$ a special lensing system. Therefore, the single gravitational lensing is a regular special lensing system.

Here we consider the problem how to construct a kind of the notion of generating families for double lensing systems. We already have a solution because a double plane gravitational lensing is described by the pair of time-delay maps. In which we only consider local properties, so that we assume that $X_{1}=X_{2}=X_{3}=\mathbb{R}^{n}$.

For any double lensing system germ $\left(S, R_{1}, R_{2}\right)$, we have generating families $F_{0}:\left(\mathbb{R}^{k_{0}} \times\right.$ $\left.X_{1}, 0\right) \longrightarrow \mathbb{R}$ of $S, F_{1}:\left(\mathbb{R}^{k_{1}} \times\left(X_{1} \times X_{2}\right), 0\right) \longrightarrow \mathbb{R}$ of $R_{1}$ and $F_{2}:\left(\mathbb{R}^{k_{2}} \times\left(X_{2} \times X_{3}\right), 0\right) \longrightarrow \mathbb{R}$ of $R_{2}$.

On the other hand, there always exists a symplectomorphism germ $\Phi_{1}:\left(T^{*} X_{1}, z_{1}\right) \longrightarrow$ $\left(T^{*} X_{1}, 0\right)$ such that $\Phi_{1}(S)$ is a zero section germ of $T^{*} X_{1}$, so that we might assume that $S$ is a zero section germ of $T^{*} X_{1}$ under the Lagrangian equivalence among double lensing system germs. In other words, it is enough to investigate special double lensing system germs. In this case $F_{0}$ can be chosen as a constant function. We call $\left(F_{1}, F_{2}\right)$ a generating pair of the special lensing system germ $\left(S, R_{1}, R_{2}\right)$ if $F_{i}$ is a generating family of $R_{i}(i=1,2)$. Then $F_{1}$ can be regarded as a generating family of $R_{1}(S) \subset T^{*} X_{2}$ and $F=F_{1}+F_{2}$ is a generating family of $R_{2} \circ R_{1}(S) \subset T^{*} X_{3}$ (cf., [42]).

Since a double gravitation lensing is a regular double lensing system, we now pay attention to regular special double lensing systems here. In this case $F_{1}:\left(X_{1} \times X_{2}, 0\right) \longrightarrow \mathbb{R}$ is a generating function of $R_{1}$ and $F_{2}:\left(X_{2} \times X_{3}, 0\right) \longrightarrow \mathbb{R}$ is a generating function of $R_{2}$. By the arguments in the previous paragraph, $F_{1}$ is a generating family of the Lagrangian submanifold germ $R_{1}(S) \subset T^{*} X_{2}$ and a map germ $F:\left(X_{1} \times X_{2}\right) \times X_{3}, 0 \longrightarrow \mathbb{R}$ defined by

$$
F\left(\left(x_{1}, x_{2}\right), x_{3}\right)=F_{1}\left(x_{1}, x_{2}\right)+F_{2}\left(x_{2}, x_{3}\right)
$$

is a generating family of the Lagrangian submanifold germ $R_{2} \circ R_{1}(S) \subset T^{*} X_{3}$. In other words, $\left(F_{1}, F_{2}\right)$ is a generating pair of a regular special double lensing system germ $\left(S, R_{1}, R_{2}\right)$ if $\left(d F_{1}\left(X_{1} \times X_{2}\right),\left(z_{1}, z_{2}\right)\right)=\left(R_{1},\left(z_{1}, z_{2}\right)\right)$ and $\left(d F_{2}\left(X_{2} \times X_{3}\right),\left(z_{2}, z_{3}\right)\right)=\left(R_{2},\left(z_{2}, z_{3}\right)\right)$. 
Since any elementary symplectic relation has a generating function at least locally, we have the following fundamental proposition:

Proposition 8.2 All regular special double lensing system germs are constructed by the above method

We can translate equivalence relations among double lensing systems into those of corresponding generating pairs.

\section{Other topics}

We describe some topics related to the singularity theory other than the topics in the previous sections.

\subsection{Ocean acoustics}

Since electromagnetic waves in the water decay very quickly, radar systems are not useful in oceans. Therefore, sonars are used as usual. In the simplest mathematical model, the wave field is governed by Helmholtz equation

$$
\left(\frac{\partial^{2} u}{\partial x^{2}}+\frac{\partial^{2} u}{\partial z^{2}}\right)(x, z)+k^{2} \eta^{2}(z) u(x, z)=f(x, z)
$$

where $\eta(z)$ is the refraction index. In general, the domain we consider has a boundary (the sea surface) and $\eta(z)$ is a piecewise smooth function. In this case, the trajectory of the sound is described by the projection of the characteristics flow of the Eikonal equation $H(x, z, p, q)=$ $\frac{1}{2}\left(p^{2}+q^{2}-\eta^{2}(z)\right)=0$ onto the $(x, z)$-plane. If $\eta(z)$ is a smooth function, we can apply our theory of graphlike unfoldings, so that the generic perestroikas of the fronts is the standard perestroikas of fronts. However, in general, $\eta(z)$ is a piecewise smooth function and we have the boundary (the sea surface) $z=0$. At the boundary point, the sound reflected following by the Snell law. These cause that we have shadow regions and broken caustics these situation do not appear in our previous framework.

There are mathematical theories for describing this rather general situation which are called the theory of the boundary caustics[69] and the theory of the reticular caustics[76]. However, these theories are not sufficient for describing our concrete case. For the previous results and the construction of the asymptotic solution for the Helmholtz equation, see [25].

\subsection{Lightlike hypersurfaces in Minkowski space}

We consider $\mathbb{R}_{1}^{3}=\left(\mathbb{R}^{3},\langle\rangle,\right)$ be Minkowski 3-space with the pseudo inner product $\langle\boldsymbol{x}, \boldsymbol{y}\rangle=$ $-x_{1} y_{1}+x_{2} y_{2}+x_{3} y_{3}$. The essential difference from the Euclidean case is the existence of the lightcone $C_{p}=\left\{\boldsymbol{x} \in \mathbb{R}_{1}^{3} \mid-\left(x_{1}-p_{1}\right)^{2}+\left(x_{2}-p_{2}\right)^{2}+\left(x_{3}-p_{3}\right)^{2}=0\right\}$. In [23] we studied singularities of the envelope of the one-parameter families of Light cones. We call such a surface a lightlike surface or a lightlike developable. In this case, the family of the lightcones always parameterized along a space like curve $\gamma: I \longrightarrow \mathbb{R}_{1}^{3}$. We may assume that the curve $\gamma(s)$ has a arc-length 
parameter (i.e., $\left\langle\gamma^{\prime}(s), \gamma^{\prime}(s)\right\rangle=1$ ). Then we have the following Frenet-Serret type formula:

$$
\left\{\begin{aligned}
\boldsymbol{t}^{\prime}(s) & =\kappa(s) \boldsymbol{n}(s) \\
\boldsymbol{n}^{\prime}(s) & =-\delta(\gamma(s)) \kappa(s) \boldsymbol{t}(s)+\tau(s) \boldsymbol{b}(s) \\
\boldsymbol{b}^{\prime}(s) & =\tau(s) \boldsymbol{n}(s)
\end{aligned}\right.
$$

where $\boldsymbol{t}(s)$ is the unit tangent vector, $\boldsymbol{n}(s)$ is the unit principal normal vector and $\boldsymbol{b}(s)$ is the unit binormal vector of $\gamma(s)$. Moreover, $\delta(\gamma(s))=\langle\boldsymbol{b}(s), \boldsymbol{b}(s)\rangle, \kappa(s)$ is the curvature and $\tau(s)$ is the torsion of $\gamma(s)$. Under the above notation, we can show that $\boldsymbol{n}(s) \pm \boldsymbol{b}(s)$ is lightlike and the lightlike surface is (at least locally) parameterized by $L D_{\gamma}^{ \pm}(s, u)=\gamma(s)+u(\boldsymbol{n}(s) \pm \boldsymbol{b}(s))$. In [18] we adopted the Lorentzian distance squared function on $\gamma$ and have shown that singularities of the lightlike surface $L D_{\gamma}^{ \pm}(s, u)$ correspond to points on $\gamma$ with the property $\left(\kappa^{\prime}(s)-\tau(s) \kappa(s)\right)=$ 0 . We have also given a classification of singularities of the lightlike surface. Moreover, we have shown that the Lorentzian invariant $\left(\kappa^{\prime}(s)-\tau(s) \kappa(s)\right)$ is constantly equal to zero if and only if the curve $\gamma$ is located on the lightcone. We can interpret that the one-dimensional analogue of the characterization for the conformal flat 3-dimensional manifold by Asperti-Dajczer[4].

We try to understand from the view point of the theory of partial differential equations. Consider the degenerate stationary Hamilton-Jacobi equation:

$$
-\left(\frac{\partial u}{\partial x_{1}}\right)^{2}+\left(\frac{\partial u}{\partial x_{2}}\right)^{2}+\left(\frac{\partial u}{\partial x_{3}}\right)^{2}=0 .
$$

The corresponding Hamiltonian function $H: T^{*} \mathbb{R}_{1}^{3} \longrightarrow \mathbb{R}$ is given by $H\left(x_{1}, x_{2}, x_{3}, p_{1}, p_{2}, p_{3}\right)=$ $-p_{1}^{2}+p_{2}^{2}+p_{3}^{2}$. The lightlike surface $L D_{\gamma}^{ \pm}(s, u)$ has a lift $\widetilde{L D} \gamma^{ \pm}(s, u)=\left(L D_{\gamma}^{ \pm}(s, u), u(\boldsymbol{n}(s) \pm \boldsymbol{b}(s))\right)$ on the cotangent bundle $T^{*} \mathbb{R}_{1}^{3}$. We can easily show that the lift $\widetilde{L D} \gamma^{ \pm}(s, u)$ is an embedding and satisfies the condition $\alpha \mid \widetilde{L D} \gamma^{ \pm}=0$. Since $\langle\boldsymbol{n}(s) \pm \boldsymbol{b}(s), \boldsymbol{n}(s) \pm \boldsymbol{b}(s)\rangle=0$, the image is contained in $H^{-1}(0)$. It follows from these facts that the lightlike surface is a solution surface (the graph of the multi-valued solution) of the equation $H=0$.

Fig.9.1 is the picture of the lightlike surface along the ellipse $\gamma(t)=(0,2 \cos t, \sin t)$ in $\mathbb{R}^{2}$.

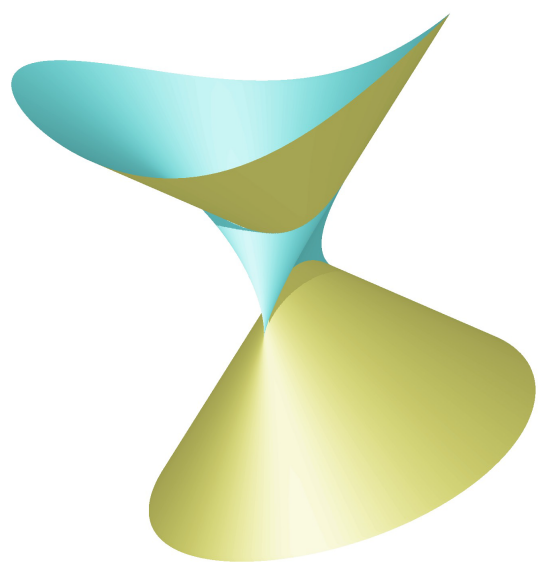

Fig. 9.1.

We can easily recognize that there exist four swallowtails. 
On the other hand, the lightlike surface is given as the tangent developable surface of a lightlike curve $\boldsymbol{\gamma}$, where $\boldsymbol{\gamma}$ is lightlike if $\dot{\gamma}(t)$ is always lightlike. In [11], we give the following classification of singularities of the lightlike tangent developable of a generic lightlike curve.

Theorem 9.1 For a "generic " lightlike curve $\boldsymbol{\gamma}$, the tangent developable of the curve $\boldsymbol{\gamma}$ at a singular point is locally diffeomorphic to $C \times \mathbb{R}, S W$ or $S B$. Here, $S B=\left\{\left(x_{1}, x_{2}, x_{3}\right) \mid x_{1}=\right.$ $\left.u, x_{2}=v^{3}+u v^{2}, x_{3}=12 v^{5}+10 u v^{4}\right\}$ is the Scherbak surface.

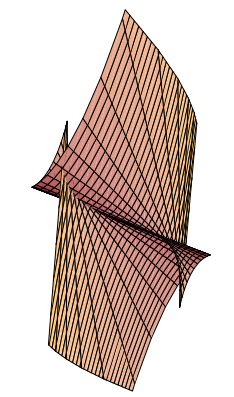

Scherbak surface

Fig.9.2.

We remark that Scherbak [70] shown that $S B$ is given as the irregular orbit of the finite reflection group $H_{3}$ on $\mathbb{C}^{3}$.

Finally we might say:

\section{There are many other singularities in the universe!}

\section{References}

[1] V. I. Arnol'd, Lagrangian manifolds with singularities, asymptotic rays, and the open swallowtail, Funct. Anal. Appl., vol 15-4 (1981), 235-246

[2] V. I. Arnol'd, S. M. Gusein-Zade and A. N. Varchenko, Singularities of Differentiable Maps vol. I. Birkhäuser (1986)

[3] V. I. Arnol'd, Geometrical Methods in the Theory of Ordinary Differential Equations, Grundlehren der mathematischen Wissenschaften 250, Springer-Verlag (1983)

[4] A. C. Asperti and M. Dajczer, Conformally flat Riemannian manifolds as hypersurfaces of the lightcone. Canad. math.Bull. 32 (1989), 281-285

[5] T. Banchoff, T. Gaffney and C. McCrory, Cusps of Gauss mappings. Research notes in Mathematics, Pitman, 55 (1982)

[6] D. E. Blair, Contact Manifolds in Riemannian Geometry. Lecture Notes in Mathematics 509 Springer (1976)

[7] D. Bleeker and L. Wilson, Stability of Gauss maps. Illinois J. Math. 22, (1978), 279-289 
[8] Th. Bröcker, Differentiable Germs and Catastrophes. London Mathematical Society, Lecture Note Series 17, Cambridge University Press, (1975)

[9] J. W. Bruce, The dual of generic hypersurfaces. Math. Scand., 49 (1981), 36-60

[10] J. W. Bruce and P. J. Giblin, Curves and singularities (second edition), Cambridge University press, (1992)

[11] S. Chino and S. Izumiya, Lightlike developables in Minkowski 3-space, preprint

[12] M.G.Crandall and P.-L.Lions, Viscosity solutions of Hamilton-Jacobi equations, Trans. AMS., 282 (1984), 487-502

[13] J.P.Cleave, The form of the tangent developable at points of zero torsion on space curves, Math.Proc.Camb.Phil. vol 88 (1980), 403-407

[14] C. G. Gibson, K. Wirthmuller, A. A. du Plessis and E. J. Looijenga, Topological stability of smooth mappings. Lecture Notes in Math., 552 Springer-Verlag, (1976)

[15] M. Golubitsky and V. Guillemin, Stable Mappings and their Singularities. Springer GTM. (1973)

[16] J. Guckenheimer, Solving a single conservation law, Lecture notes in Mathematics 468 Springer Verlag, New York, (1975), 108-134

[17] J. Hoschek and H. Pottman, Interpolation and approximation with developable B-spline surfaces, in Mathematical Methods for curves and surfaces, ed. by M. Dæhlen, T. Lyche and L.L. Schumacker, Vanderbilt Univ. Press (1995), 255-264

[18] G. Ishikawa, Determinacy of envelope of the osculating hyperplanes to a curve, Bull.London Math.Soc., vol 25 (1993), 787-798

[19] G. Ishikawa, Developable of a curve and determinacy relative to osculation-type, Quart. J. Math. Oxford, vol 46 (1995), 437-451

[20] S.Izumiya and G.T.Kossioris, Geometric singularities for solutions of single conservation laws, Arch. Rat. Mech. Anal., 139 (1997), 255-290

[21] S. Izumiya and T. Sano, Generic affine differential geometry of space curves, Proceedings of the Royal Society of Edinburgh , vol 128A (1998), 301-314

[22] S. Izumiya, H. Katsumi and T. Yamasaki, The Rectifying developable and the Darboux indicatrix of a space curve, Geometry and Topology of Caustics-Caustics '98, Banach Center Publications, vol 50 (1999), 137-149

[23] S. Izumiya, D.-H. Pei and T. Sano, The lightcone Gauss map and the lightcone developable of a spacelike curve in Minkowski 3-space, Glasgow Math. J., vol 42 (2000), 75-89

[24] S. Izumiya and N. Takeuchi. Singularities of ruled surfaces in $\mathbb{R}^{3}$. Math. Proceedings of Cambridge Philosophical Soc.,vol 130 (2001),1-11

[25] S.Izumiya, G.Kossioris and G. Makrakis: Multi-valued solutions to the eikonal equation in stratified media, Quarterly of Applied math. LIX, (2001) 365-390 
[26] S.Izumiya and N. Takeuchi. Generic properties of Helices and Bertrand curves. Journal of Geometry, 74, (2002), 97-109

[27] S. Izumiya and N. Takeuchi. Special curves and ruled surfaces. Beiträge zur Algebrra und Geometrie, 44 (2003), 203-212

[28] S. Izumiya and N. Takeuchi. New special curves and developable surfaces. Turk. J. Math. 28 (2004),153-163

[29] S. Izumiya, D-H. Pei and T. Sano, Singularities of hyperbolic Gauss maps. Proceedings of the London Mathematical Society 86 (2003), 485-512

[30] S. Izumiya and S. Janeczko, A symplectic framework for multiplane gravitational lensing, Journal of Mathematical Physics 44, (2003) 2077-2093

[31] S. Izumiya and M. C. Romero-Fuster, The horospherical Gauss-Bonnet type theorem in hyperbolic space, to appear in Journal of Mathematical Society of Japan

[32] S. Izumiya, D-H. Pei, T. Sano and E. Torii, Evolutes of hyperbolic plane curves, Acta Mathmatica Sinica 20, (2004), 543-550

[33] S. Izumiya, D-H. Pei and T. Sano, Horospherical surfaces of curves in Hyperbolic space, Publ. Math. (Debrecen) 64 (2004),1-13

[34] S. Izumiya, D-H. Pei and M. Takahasi, Curves and surfaecs in Hyperbolic space, Banach center publications 65, Geometric singularity theory (2004), 197-123

[35] S. Izumiya, D-H. Pei, M. C. Romero-Fuster and M. Takahashi, On the horospherical ridges of submanifolds of codimension 2 in Hyperbolic n-space, Bull. Braz. Math. Soc. 35 (2) (2004), $177-198$

[36] S. Izumiya, D-H. Pei and M. Takahashi, Singularities of evolutes of hypersurfaces in hyperbolic space, Proceedings of the Edinburgh Mathematical Society 47 (2004), 131-153

[37] S. Izumiya, D-H. Pei and M. C. Romero-Fuster, The horospherical geometry of surfaces in Hyperbolic 4-space, to appear in Israel Journal of Mathematics

[38] S. Izumiya, M. C. Romero-Fuster, D-H. Pei and M. Takahashi, Horospherical geometry of submanifolds in hyperbolic n-space, Journal of London Mathematical Society 71, (2005) 779800

[39] S. Izumiya, Differential geometry from the viewpoint of Lagrangian and legendrian singularity theory. To appear in the proceedings of Luminy conference.

[40] S. Izumiya, Legendrian dualities and spacelike hypersurfaces in the lightcone, preprint

[41] S. Izumiya and M. Takahashi, Spacelike parallels and evolutes in Minkowski pseuod-spheres, preprint

[42] S. Janeczko, Generating families for images of Lagrangian submanifolds and open swallowtails, Mathematical Proceedings of Cambridge Phil. Soc. vol. 100 (1986),91-107

[43] J. J. Koenderink, What does the occluding contour tell us about solid shape? Perception, 13 (1984), 321-330 
[44] J. J. Koenderink, Solid shape, The MIT Press, Cambridge, Massachusetts, (1990)

[45] E. E. Landis, Tangential singularities, Funct. Anal. Appli., 15 (1981), 103-114

[46] H. I. Levine, A.O. Petters, J. Wambsganss, Applications of singularity theory to gravitational lensing I, Multiple lens planes, J. Math. Phys. vol. 34 (1993),4781-4808

[47] H. I. Levine and A. O. Petters, New caustics singularities in multiple lens plane gravitational lensing, Astron. Astrophys. vol. 272 (1993),L17-L19

[48] J. Martinet, Singularities of Smooth Functions and Maps, London Math. Soc. Lecture Note Series, Cambridge Univ. Press,58 (1982)

[49] J.N. Mather, Stability of $C^{\infty}$-mappings I:The division theorem, Ann. of Math., 87 (1968), 89-104,

[50] J. Mather, II:Infinitesimally stability implies stability, Ann. of Math., 89 (1969), 254-291,

[51] J. Mather, III:Finitely determined map-germs, Publi. Math. I.H.E.S., 35 (1968), 127-156.

[52] J. Mather, IV:Classification of stable germs by $\mathbb{R}$ algebras, Pub.. Math. I.H.E.S., 37 (1970), 223-248.

[53] J. Mather, V:Transversality, Adv. Math., 4 (1970), 301-336.

[54] J. Mather, VI:The nice dimensions, Lecture Notes in Math., 192 (1972), 207-253

[55] J. Milnor, Morse theory, Ann. Math. Stud. 51, Princeton University Press (1963)

[56] D. Mond, On the tangent developable of a space curve, Math. Proc. Cambridge Phil. Soc. 91 (1982), 351-355.

[57] J. A. Montaldi, Surfaces in 3-space and their contact with circles, J. Diff. Geom., 23 (1986),109-126

[58] J. A. Montaldi, On contact between submanifolds, Michigan Math. J., 33 (1986), 81-85

[59] J. A. Montaldi, On generic composites of maps, Bull. London Math. Soc., 23 (1991), 81-85

[60] O. Oleinik, Discontinuous solutions of nonlinear differential equations, Amer. Math. Soc. Transl. Ser. 2, 26 (1957) 95-172

[61] A. O. Petters, Arnol'd's singularity theory and gravitational lensing, J. Math. Phys. vol. 34 (1993),3555-3581

[62] A.O.Petters, Multiplane gravitational lensing I, Morse theory and image counting, J.Math. Phys. vol. 36 (1995), 4263-4275

[63] A.O.Petters, H.Levine and J. Wambsganss, Singularity theory and Gravitational lensing, Birkhäusr (2001)

[64] A. A. du Plessis and C. T. C. Wall, The geometry of topological stability, Oxford University Press, (1995)

[65] I. Porteous, The normal singularities of submanifold, J. Diff. Geom., vol 5, (1971), 543-564 
[66] I. Porteous, Geometric Differentiation second edition, Cambridge Univ. Press (2001)

[67] M. C. Romero Fuster, Sphere stratifications and the Gauss map. Proceedings of the Royal Soc. Edinburgh, 95A (1983), 115-136

[68] T. Sasaki, Projective Differential Geometry and Linear Homogeneous Differential Equations, Rokko Lectures in Mathematics, Kobe University, vol 5 (1999)

[69] I. G. Scherbak, Boundary fronts and caustics and their metamorphosis, London Math. Soc. Lecture note series 201, Singularities, (1994), 363-373

[70] O.P.Scherbak, Wavefront and reflection groups, Russian Math. Surveys 43-3 (1988), 149-194.

[71] O.P.Scherbak, Projectively dual space curves and Legendre singularities, Trudy Tbiliss. Univ. 232-233 (1982), 280-336.

[72] P.S.Schneider, J.Ehlers and E.E. Falco, Gravitational lenses, Springer (1992)

[73] M. Schneider, Interpolation with Developable Strip-Surfaces Consisting of Cylinders and Cones, in Mathematical Methods for curves and surfaces II, ed. by M. Dchlen, T. Lyche and L.L. Schumacker, Vanderbilt Univ. Press, (1998), 437-444

[74] R. Thom and H. Levine, Singularities of differentiable mappings, Bonner Mathematische Schriften, vol 6, Bonn, (1959)

[75] J. A. Thorpe, Elementary Topics in Differential Geometry, Springer-Verlag

[76] T. Tsukada, Reticular Lagrangian singularities, Asian J. of Math., 1 (1997), 572-622

[77] I. Vaisman. A first course in differential geometry. Pure and applied Mathematics,A series of Monograph and Textbooks, MARCEL DEKKER, (1984)

[78] H. Whitney, The singularities of a smooth n-manifold in $(2 n-1)$-space, Annals of Math., vol 45 (1944), 247-293

[79] V. M. Zakalyukin, Lagrangian and Legendrian singularities, Funct. Anal. Appl., 10 (1976), $23-31$

[80] V. M. Zakalyukin, Reconstructions of fronts and caustics depending one parameter and versality of mappings. J. Sov. Math., 27 (1984), 2713-2735

Shyuichi Izumiya, Department of Mathematics, Hokkaido University, Sapporo 060-0810,Japan e-mail:izumiya@math.sci.hokudai.ac.jp 